\title{
High Power Ultrasonics in Pyrometallurgy: Current Status and Recent Development
}

\author{
Sergey V. KOMAROV, Mamoru KUWABARA ${ }^{11}$ and Oleg V. ABRAMOV ${ }^{21}$ \\ IMRAM, Tohoku University, 2-1-1 Katahira, Aoba-ku, Sendai 980-8577 Japan. E-mail: komarov@tagen.tohoku.ac.jp \\ 1) Department of Materials Processing Engineering, School of Engineering, Nagoya University, Furo-cho, Chikusa-ku, Nagoya \\ 464-8603 Japan. E-mail: kuwabara@numse.nagoya-u.ac.jp $\quad 2$ 2) Institute of General and Inorganic Chemistry, Russian \\ Academy of Sciences, Leninsky prospect 31, 117907, Moscow, Russia. E-mail: LUTT@igic.ras.ru
}

(Received on July 11, 2005; accepted on August 26, 2005)

\begin{abstract}
In recent years, a large number of studies have been published on the use of high intensity ultrasonics in various high temperature technologies. This paper provides an overview of the recent achievements and ongoing works on the application of high intensity sound waves to pyrometallurgy and its related areas. The published results have strongly suggested that ultrasonics has the potential to play a more significant role in such areas as the dedusting of high-temperature exhaust gas, improvement of fuel-combustion efficiency, control of air-pollutant emissions, improvement of the quality of ingots, production of metal powders and ascast composite materials.

At higher temperatures, special attractiveness of sound waves is associated with the fact that the waves can propagate through gas, liquids, and solids, and thus supply the acoustic energy from a cooled sonic generator to materials being processed under high temperature conditions. This provides a unique tool, for example, for controlling the rates of interfacial phenomena that is unachievable by any other methods under high temperatures.

Industrial competitiveness of the ultrasonic-based technologies is reinforced by the relatively low cost of power-generating equipment and ultrasonic transducers. However, further research efforts are called for to develop new heat-resistant waveguide materials and to integrate the ultrasonic installations with existing industrial facilities in high temperature technologies.
\end{abstract}

KEY WORDS: pyrometallurgy; high temperature; sonoprocessing; high power ultrasonics; non-linear phenomena; air pollutants; continuous casting; melt atomization; cast composites.

\section{Introduction}

The idea of using ultrasonic oscillations for improving process performance or for changing material structure is rather old. As early as the 1920 s, Wood and Loomis ${ }^{1)}$ had studied the effects of ultrasonics on the atomization of liquids, the emulsification of immiscible liquids, and the changes in the structure of crystallized organic substances.

In the years that followed, ultrasonic effects have become the subject of numerous extensive studies. The studies have proved the benefits of using ultrasonics in such fields as the chemical and food industries, biology, and medicine. The results of these studies are all around us. The ultrasound is so widely used in various spheres of activity that their thorough enumeration would take much time.

The effects of ultrasonics are associated with the ability of sound to propagate through such elastic mediums as gas, liquids, and solids, and thus to transfer the acoustic energy from a sonic generator to the materials being processed. Although both the sound generation and propagation are influenced by many factors, the most important condition for the effective supply of acoustic energy is the ability to provide a reliable contact between the oscillating parts of the sonic generator and the elastic medium. In pyrometallurgical applications, where high temperatures are involved, creating this condition may incur significant difficulties, because it requires designing a cooling system, choosing special materials for the waveguide components, and optimizing them under conditions of sharp temperature drops. Despite this, the first attempt to apply ultrasonics to metallurgical processes was made long ago, a few years after the experiments of Wood and Loomis. The idea belongs to Sokolov, 2,3) who worked at the University of Saint Petersburg. He is said to be the father of ultrasonic testing, though he also conducted a number of studies on the crystallization of metals under conditions of ultrasonic irradiation.

Since that time, a great number of studies, both fundamental and applied, have been performed on the use of ultrasonics in metallurgy and other high-temperature processes. The results have revealed that high-intensity ultrasonics could be used in such areas as the dedusting of high-temperature exhaust gas, the degassing of liquid metals, the improvement of the quality of ingots, the production of metal powders, the enhancement of heat and chemicothermal treatment rates, metalworking, and welding. Some of these 
applications have been summarized in a book by one of the authors of the present review. ${ }^{4)}$

The following two circumstances make ultrasonics especially applicable to high-temperature technologies:

1) There is a severely limited choice of techniques available for supplying energy under conditions involving higher temperatures. Among these techniques, sonic/ ultrasonic treatment or sonoprocessing should be competitive as regards both technique and cost, because it provides an effective transmission of acoustic energy from the ultrasonic generators to the materials being processed at a relatively low cost for ultrasonic equipment.

2) It is well-known that interfacial phenomena play an important role in governing many high-temperature processes. Examples are mass and heat transfer, crystal growth during the solidification of liquid metals, wetting, and emulsification. Sonic and ultrasonic waves propagate through homogeneous elastic mediums without significant losses. However, when the waves are incident upon an interface, the scattering or reflecting or of the waves from the interface is responsible for a number of nonlinear phenomena that occur at the interfaces. These provide a unique tool for controlling the rates of interfacial phenomena. Such a tool is unachievable by any other methods.

Along with improvements in the earlier methods of sonoprocessing, a number of new ultrasonic applications have been found in areas yet unexplored that bear on metallurgy and metal working. Among these are the improvement of fuel-combustion efficiency, control of air-pollutant emissions, production of as-cast composite materials, thixocasting, surface metallization, cleaning of metal-roll production, and the degreasing of mill scale.

The goal of this paper is to review recent achievements and ongoing works on the application of ultrasonics to pyrometallurgy and its related high-temperature processes. It is to be noted that even in pyrometallurgy and related areas, sonoprocessing is a very wide topic that cannot be covered in one review. In this connection, we restrict our review with the following two criteria. First, all studies reviewed have either a direct or an indirect relationship to ferrous metallurgy. Some of the sonic and ultrasonic applications have been tested on the appropriate metallurgical units, while the others have been examined by using pilot or laboratory scale equipment, and are potentially applicable to processes in ferrous metallurgy. Second, the present review is restricted to those applications that use either gas or liquid as an elastic medium for transferring acoustic energy. That is the reason that many interesting applications, based on ultrasonic propagation through solid phases, remain beyond the scope of this review. Of note among these are ultrasonic welding, heat treatment, metalworking, plating, and machining. Each is worthy of review.

\section{Brief Theoretical Background}

\subsection{General Characteristics of Traveling Sound Waves}

In processes which use the energy of sound or ultrasound waves, a necessary condition for obtaining desired effects is to provide enough high intensity of the waves. Propagation of a high intense sound wave causes such non-linear phenomena as cavitation, acoustic streaming and radiation pressure. These phenomena are the basis for the most of ultrasonic effects presented in this review. This section introduces some important characteristics of sound waves and, then, briefly consider the non-linear effetcs to that extent which is necessary for understanding the matter of the present paper.

When a wave propagates through an unbounded medium, the wave is said to be traveling. In a traveling wave, the oscillatory motion can be expressed in terms of displacement of the particle relative to their equilibrium position as follows

$$
\xi=\xi_{0} \sin (\omega t-k x)
$$

This equation is the basis for derivation of other equations which describe the wave propagation. Thus, the velocity of oscillations, $V$, and pressure, $P$ can be given as

$$
\begin{aligned}
& V=V_{0} \cos (\omega t-k x) . \\
& P=P_{0} \sin (\omega t-k x) .
\end{aligned}
$$

where $\omega=2 \pi f$ and amplitudes $\xi_{0}, V_{0}, P_{0}$ are related to each other as $V_{0}=\xi_{0} \omega$ and $P_{0}=\xi_{0} \omega \rho c$. The wave number $k$ can be simply defined as $k=\omega / c$ or as $k=2 \pi / \lambda$. Frequency, $f$ is commonly subdivided into audible sonic and inaudible ultrasonic ranges although physically there is no difference between sonic and ultrasonic waves. The boundary between these ranges lies at about $16 \mathrm{kHz}$.

Based on the above amplitude characteristics, one can derive an expression of sound intensity, $J$, which is defined as the average rate of sound energy transmitted by a travelling wave through a unit area normal to the direction of sound propagation

$$
J=\frac{1}{2} \rho c V_{0}^{2}
$$

In practice, since the sound intensity varies by several orders of magnitude, it is often expressed in logarithmic form known as sound intensity level, SIL

$$
S I L=10 \log \frac{J}{J_{\text {ref }}}
$$

where the reference intensity, $J_{\text {ref }}$ is equal to $10^{-12} \mathrm{~W} / \mathrm{m}^{2}$. The unit of SIL is decibel, $d B$.

Although there is no universally accepted definition of low and high-intense sound wave, it is clear that any wave to be considered as a high-intense one must have amplitude greater than some threshold value. In practice, this value is most commonly estimated on the basis of whether the wave is able to produce the above mentioned nonlinear effects. For liquids including molten metals, the threshold value of $V_{0}$ can be determined from the following conditions ${ }^{4)}$

$$
M a=\frac{V_{0}}{c}>10^{-4}
$$

Since for the most of molten metals, $c$ is ranged from 1600 to $5000 \mathrm{~m} / \mathrm{s},{ }^{5-7)}$ the velocity amplitude must be larger than $0.16-0.5 \mathrm{~m} / \mathrm{s}$ in order to produce high-intense sound 
wave. For gases, the ratio of $V_{0}$ to $c$, it seems, must be an order greater. ${ }^{8,9)}$

\subsection{Attenuation of Sound Waves}

As a sound wave is propagated through a medium, its intensity is reduced or attenuated through a number of mechanisms including divergence, scattering and energy dissipation. Divergence is the most simple phenomenon among the above-mentioned list. It results from a geometric spreading of the sound wave occurring with a loss of the wave intensity proportional to the square of the distance from the sound source.

Scattering refers to the reflection of the incident sound wave from an obstacle with dimension close to or less than the sound wavelength. Here, by an obstacle is meant any foreign substance on the second phase, for instance particulate or bubble, acoustic impedance of which differs greatly from that of the surrounding medium. Acoustic impedance, $Z$ is defined as the product of density, $\rho$ by sonic velocity, $c$.

$$
Z=\rho c \text {. }
$$

Despite the fact that mechanism of scattering is very complicated, different theoretical considerations and experimental observations suggest similar relationships between the intensity of scattering and three key parameters: sound frequency, $f$, obstacle dimension, $d$ and its mass fraction, $C_{\mathrm{o}}$. Thus, Landau and Lifshits ${ }^{10)}$ derived a formula which suggests that the intensity of scattering, $I_{\mathrm{s}}$ is proportional to the sixth power of $d$ and fourth power of $f$ for the case of rigid particles suspended in a gas. The reported dependences of $I_{\mathrm{s}}$ on $C_{\mathrm{o}}$ are linear for both the gaseous ${ }^{11)}$ and liquid mediums. ${ }^{12)}$ Therefore, the intensity of scattering becomes more or less significant only at higher frequencies, generally in the $\mathrm{MHz}$ range and at larger mass fractions of particulates or bubbles. It is to be noted that the scattering is of fundamental importance in governing the radiation pressure discussed below.

Sound energy dissipation, or absorption, is assumed to be the main cause of sound intensity attenuation in most of the applications reviewed below. Generally, there are two mechanisms of sound energy absorption in fluids. The viscous dissipation occurs due to the normal and shear stresses induced in fluids on sound wave propagation. The dissipation due to heat conduction results from non-adiabatic nature of heat transfer during compression and rarefaction cycles in sound wave. Both these mechanism contribute the sound energy dissipation that can be expressed quantitatively in terms of the absorption coefficient, $\alpha$, that is the sum of the viscosity-related term, $\alpha_{V}$, and heat conduction-related term, $\alpha_{T}$. For low-amplitude oscillations, $\alpha$ is expressed by Eq. (8)

$$
\alpha=\alpha_{V}+\alpha_{T}=\frac{\omega^{2}}{2 \rho c^{3}}\left(\frac{4}{3} \eta+\eta^{\prime}\right)+\frac{\omega^{2}}{2 \rho c^{3}} \kappa\left(\frac{1}{C_{V}}-\frac{1}{C_{P}}\right)
$$

where $\omega=2 \pi f, \eta$ is the shear viscosity, $\eta^{\prime}$ is the bulk viscosity, $\kappa$ is the thermal conductivity, $C_{V}$ and $C_{P}$ are the specific heats at constant volume and pressure. In this case, attenuation of sound intensity due to absorption follows the exponential law according to Eq. (9).

$$
J_{x}=J_{0} e^{-2 \alpha x}
$$

where $J_{0}$ and $J_{x}$ are the intensities of sound waves at two points spaced each other by a distance of $x$ in the direction of wave propagation. As readily seen from Eq. (8), energy absorption varies in proportion to the square of the frequency, $f$.

Attenuation of high-amplitude sound oscillations occurs more vigorously than that of low-amplitude oscillations. The stronger attenuation is associated with the conversion of a part of initial sinusoidal wave into the energy of arising high-frequency harmonics, rather than with absorption energy. Therefore, the attenuation coefficient of high-amplitude sound wave can exceed the absorption coefficient of low-amplitude sound wave by two orders of magnitude. There are also the other reasons but their detailed consideration is beyond the scope of this review. The relevant information on this topic can be found in books. ${ }^{4,13)}$

\subsection{Standing Wave}

When a sound wave is incident normally upon a flat surface of a body which fully reflects the wave, interference between incident and reflected waves may results in formation of a standing wave. The reflection occurs provided the acoustic impedance of a medium where the wave propagates is significantly different from that of the body. This condition is fulfilled for gas-liquid, gas-solid and liquidsolid interfaces almost without exception. Such a wave is characterized by the existence of nodes and antinodes that are fixed in space.

In standing wave, contrary to travelling one, there is no energy transfer. Instead, in standing wave, the energy is alternately transferred from kinetic energy to potential energy. By analogy with sound intensity level, SIL, the energy inside a standing wave can be evaluated by the sound pressure level, $S P L$ in $\mathrm{dB}$ units as follows

$$
S P L=20 \log \frac{P_{0}}{P_{\text {ref }}}
$$

here the reference pressure, $P_{\text {ref }}$ is equal to $2 \times 10^{-5} \mathrm{~Pa}$.

A special case occurs when a standing wave is formed between parallel surfaces, one of which is a sound radiator while the other one is a reflecting surfrace, provided that the distance between these two surfaces, $L$ is an integer multiple of one-half wavelength according to Eq. (11)

$$
L=\frac{n \lambda}{2}, \quad n=1,2,3, \ldots
$$

This kind of wave, called resonance standing wave, is very important for practical application because it allows obtaining very high levels of sound pressure in the treated medium under relatively moderate energy suppy. In practice, geometry of reactors is much complicated than the above parallel surfaces. Therefore, the resonance conditions are determined experimentally by measuring the sound pressure inside the reactor as a function of the sound frequency and reactor geometric parameters. A resonance condition corresponds to that condition under which a maxi- 
mum value of pressure was detected.

\subsection{Acoustic Nonlinear Effects}

As noted above, the sound energy attenuation is responsible for a number of non-linear effects which serve as the basis for many ultrasonically based technologies.

The acoustic nonlinear effects arising in fluid-containing systems are summarized in Table $\mathbf{1}$.

There is a great body of literature devoded to both the theoretical and experimental investigations of the above effects. Detailed discussion on the results of these investigations can be found in a variety of books, e.g. ${ }^{13-16)}$ and reviews, e.g. ${ }^{17-19)}$ This section briefly outlines only those phemomena which are of primary importance in understanding the matter presented in the following sections.

\subsubsection{Cavitation}

Cavitation is the phenomenon giving rise to formation of tiny discontinuities and cavities in a liquid followed by their growth, pulsation and collapse. The cavities appear as a results of the tensile stress produced by an acoustic wave in its rarefaction phase. To disrupt a liquid and produce a cavity, it is necessary that the amplitude of acoustic pressure be above some critical value which is termed the cavitation threshold, $P_{\mathrm{c}}$. In ideal liquids, $P_{\mathrm{c}}$ is governed by molecular forces only. But, in practice, the presence of solid particles, vapor and gaseous microbubbles in liquids results in a significant reduction of the cavitation threshold compared to the ideal case. It is found that the bigger the microbubble size, the lower is the the cavitation threshold. Effect of solid particles on $P_{\mathrm{c}}$ varies with their size, shape and wettability. According to the reported results, a solid nonwettable particle with cracks filled with an insoluble gas decreases $P_{\mathrm{c}}$ serving as a cavitation nucleus. ${ }^{20)}$ This finding is especially important for liquid metals, usually containing various nonmetallic inclusions. Another factor influencing $P_{\mathrm{c}}$ is concentration of solid particles. Experimental observations revealed that the cavitation threshold of molten $\mathrm{Al}$ decreases as the content of $\mathrm{Al}_{2} \mathrm{O}_{3}$ particles, introduced into the melt, increases. ${ }^{21)}$ Besides, $P_{\mathrm{c}}$ tends to increase with frequency, when the frequency exceeds a certain value. $\left.{ }^{4}\right)$ For the most system examined this value is about $25 \mathrm{kHz}$. The experimentally observed threshold cavitation pressure in liquid metals is ranged from about 0.25 to $0.9 \mathrm{MPa}$.

Once a cavity has formed, its further behaviour is conditioned by both the properties of liquid and characteristics of acoustic field. During cavitation, many cavities appear simultaneously at distances less that the wavelength to form a cavitation region of very high-density energy inside and near collapsing cavities. The rate of cavity collapse can be so high that it results in emission of a high-pressure shock which, in turn, can cause formation of microjets along the wave propagation. This ultrasonically induced microjetting is the origin of most of the ultrasonic effects discussed in the present review with reference to processes dealing with liquid metals. It was shown that the amplitude of shock waves is large only near the collapsing cavity, being decreased inversely with the distance from it. ${ }^{22}$ This explains why cavitation-related effects are most pronounced near collapsing cavities.

In addition to the formation of shock waves, the bubble
Table 1. Acoustic nonlinear effects.

\begin{tabular}{l|l}
\hline \multicolumn{1}{c|}{ Medium, system } & \multicolumn{1}{c}{ Effect } \\
\hline Gas & Acoustic streaming, vorticity \\
Liquid & Cavitation, acoustic streaming \\
Gas-Liquid Interface & Capillary waves, atomization \\
Liquid-Liquid Interface & Capillary waves, emulsification \\
Gas-Solid Interface & Acoustic streaming, radiation pressure \\
Liquid-Solid Interface & Acoustic streaming, radiation pressure, \\
& microjetting, shock stress \\
\hline
\end{tabular}

collapse results in a drastic increase in temperature inside the bubble. Calculations yielded the values of about $10000 \mathrm{~K}$ for water $^{23)}$ and $5000 \mathrm{~K}$ for silicon oil. ${ }^{24)}$

\subsubsection{Acoustic Streaming}

Acoustic streaming is the steady flow that is generated in a fluid medium due to momentum transfer associated with the attenuation of a sound wave. Physically, the origin of acoustic streaming was best explained by J. Lighthill. ${ }^{15)}$ Briefly, the explanation is as follows. Propagation of a sound wave causes fluctuations of the medium fluid particles at certain time scale and amplitude which are dictated by the wave frequency and intensity, respectively. By applying the Reynolds stress approach to express the mean momentum flux due to the wave in the same manner as it does for turbuent pulsations, one can derive Reynolds stress, $\tau$, in a sound wave as

$$
\tau=\overline{\rho u_{i} u_{j}}
$$

where $u_{i, j}$ are the fluctiating velocity in the sound wave and the bar signifies a mean value. The wave attenuation results in a spatial variaton of the Reynolds stress that can cause a non-zero net force exerted on the fluid. This force is capable of generating a steady flow termed acoustic streaming. It is generally recognized that there three types of acoustic streamings. They differ each other by the spatial scale on which they can spread. The acoustic streaming of the first type is generated in an unbounded body of fluid. As its scale is much larger compared to the sound wavelength, it is called large-scale streaming. In this case, the streaming originates from the sound energy absorption briefly considered above. Because the attenuation coefficient increases with the square of the sound frequency, the large-scale streaming becomes significant only at high frequencies.

The acoustic streamings of the second and third types are generated in the presence of solid obstacles (walls, particulates) placed in an acoustic field. In this case, the attenuation occurs because of frictional dissipation between an oscillating fluid and solid surface within the resulting boundary layer.

The acoustic streaming of the second type is generated outside the boundaly layer. The scale of such an outer streaming is much smaller than that of the first type, and is equal approximately to the wavelength. The acoustic streaming of the third type is called inner small-scale streaming because it is induced within the bounday layer, the dimension of which is much smaller than wavelength. The effective thickness of the boundaly layer is about 5 times larger than that of acoustic boundaly layer, $\delta=$ $(v / \omega)^{0.5}$, where $v$ is the kinematic viscosity of fluid, $\omega$ is 
the angular frequency of sound, $\omega=2 \pi f .{ }^{19)}$

All these three types of acoustic streaming play an important role in controlling many physical and chemical phenomena in fluids being processed ultrasonically although the control mechanism seems to be different. While the large-scale acoustic streaming promotes macromixing in the gas or liquid bulk, the other two types, especially, the small-scale acoustic streaming enhances micromixing at near-interface regions. This makes the acoustic streaming, along with cavitation, an unique tool in controlling, for example, structure of solidifying metals. Another example where high-intense sonic and ultrasonic waves are of particular assistance is processes the rates of which are controlled by gas/liquid phase heat or mass transfer. Here, acoustic oscillations provide an effective means for perturbation of the boundary layer that can significantly enhance the heat and mass transfer rates.

\subsubsection{Acoustic Radiation Pressure}

Another interesting phenomenon arising in high-intense acoustic fields is radiation pressure. The radiation pressure is produced by a non-uniformity of sound field around an obstacle positioned in the field. As a result, the average pressure exerted on the obstacle is not 0 . This yields a steady force acting on the obstacle. Notice that the term obstacle here implies not only real objects like solid particles in a gas or bubbles in a liquid, but also different volumes of the same medium. Such a situation can occur when a sound wave propagates through an unbounded medium and attenuates due to dissipation during its propagation. Another example is the propagation of sound through a gaseous medium with a large temperature gradient like flame.

It should be noted that radiation pressure is very weak in comparison to sound pressure. Hence, the radiation pressure can be significant only in very intense acoustic fields. However, in some special cases, the radiation pressure provides a means to obtain those effects which can not be achieved by the other methods.

For the present review, of prime interest is to briefly consider the effect of radiation pressure on objects suspended in gas or liquid. In this case, the acoustic field non-uniformity is resulted from scattering or reflection of sound waves from the object surfaces. The radiation pressure can exert a rotating action on an asymmetrically shaped object such as a rod or disc suspended in a liquid. This acoustically induced torque may vary the orientation of the object relative to the oscillation direction of the acoustic fileld, so that the object tends to take the position in which the torque on the object is least. Such an effect can be exploited, for example, to produce a composite material with matrix and unidirectionally oriented reinforcing elements.

For a symmetrical object such as a sphere, the radiation pressure can force the object to move in the direction of applied acoustic field. For the simplest case, when a imcompressible spherical particle is positioned on the path of a travelling plane wave the force, $F_{\mathrm{t}}$ acting on the particle can be expressed as follows ${ }^{8)}$

$$
F_{\mathrm{t}}=\frac{11}{9} \pi R^{2}(k R)^{4} \bar{E}
$$

In the case of a standing wave, the radiation force can, $F_{\mathrm{s}}$ be written as ${ }^{8)}$

$$
F_{\mathrm{s}}=\frac{8}{3} \pi R^{2}(k R) \bar{E} \sin 2 k x_{0}
$$

where, $R$ is the particle radius, $k$ is the wave number, $x_{0}$ is the distance between particle and the nearest displacement node in the standing wave, $\bar{E}$ is the acoustic enegry density $(=J / c), J$ is the acoustic field intensity.

A comparison of Eqs. (13) and (14), in view of small $k R$, makes it clear that radiation pressure in standing wave can reach much greater values than those in travelling wave. Another important conclusions to be made is that radiation pressure increases with the particle radius and frequency of acoustic field. Tha latter is because the wave number, $k$, is proportional to wave frequency. For the more details, the reader is referred to the appropriate publications. ${ }^{4,25)}$

\section{Devices and Techniques for Sonoprocessing}

The industrial ultrasonic facility includes a transducer converting a particular type of energy (electric or mechanical) into acoustic energy, power supply, radiator, and various systems for providing measurement and control of operating parameters. Although higher temperatures of pyrometallurgical processes impose limitations on the design of some parts of the ultrasonic equipment, generally sonic/ultrasonic high temperature installations are based on the well-known principles in ultrasonic engineering. First of all, the sonic/ultrasonic installations are divisible into two big groups depending on what principle of energy conversion they use $\mathrm{e}^{4)}$ :

1) Electroacoustic systems

2) Mechanoacoustic systems.

\subsection{Electroacoustic Systems}

Electroacoustic systems are based on transducers which convert energy of electromagnetic field into energy of acoustic oscillations. These transducers can be subdivided into those based on electrodynamic, piezoelectric or magnetostrictive effects. The electrodynamic transducer operates by the similar principle as a loudspeaker converting an alternating electrical signal into the corresponding magnetic field, and then into mechanical oscillations of a cone attached to a wire coil. Although acoustic power generated by the electrodynamic transducers is relatively low, their great advantage is that they allow the sound frequency to adjust to a desired value, for example, for obtaining resonance conditions. That is why the electrodynamic transducers have found a practical use in experimental and pilot scale equipment to transmit acoustic energy through a gas atmosphere, e.g. ${ }^{70)}$ As a rule, the electrodynamic transducers are used within a low frequency range from 20 to $2000 \mathrm{~Hz}$.

The operating principle of piezoelectric transducers is based on the so-called reverse piezoelectric effect that consists in the production of a strain in some anisotropic insulators and semiconductors under application of an electric field, e.g. ${ }^{26)}$ The piezoelectric transducers have found a very wide practical use in many industrial applications due to a number of advantages. Among them are the high (up to 
$80 \%$ ) electroacoustic efficiency, wide band of operating frequencies ranging from a few $\mathrm{kHz}$ to tens of $\mathrm{MHz}$, relatively low prices for piezoelectric equipment. Nevertheless, the piezoelectric transducers are of limited application for high temperature processes because of difficulties in cooling the transducer parts, brittleness of piezoelectric materials and their short life.

Magnetostrictive transducers are the most promising device for ultrasonic application under higher temperatures. Magnetostriction is defined as a relative change in linear dimension of a ferromagnetic body with respect to its initial length during magnetization. Physically, magnetization results from interaction between an external magnetic field and magnetic domains of the body. In such ferromagnetic materials as $\mathrm{Fe}, \mathrm{Ni}, \mathrm{Co}$, in a number of alloys and ferrites the magnetostriction can achieve significant values, up to 0.01 . $^{4}$ The simplest magnetostrictive transducer represents a ferro- or ferrimagnetic core inside a coil through which an alternating current flows to create a magnetic field. Magnetization of the core gives rise to alternating elastic stresses and strains directed axially. As a result, the core begins to vibrate longitudinally.

The electroacoustic efficiency of magnetostrictive transducers is a little less than that of piezoelectric transducers. Thus, the efficiency of transducers operated within a frequency range of $0-20 \mathrm{kHz}$, is $40-50 \%$ and diminishes as the working frequency increases. ${ }^{4}$ However, compared to the piezoelectric transducers, they provide higher mechanical properties, more simple and effective solutions in development of cooling systems, possibilities for designing very powerful and compact modules.

In the same manner as piezoelectric transducers, a magnetostrictive transducer operates at a resonance frequency. Therefore, dimensions of all vibrating parts of transducer are rigidly dependent on the required working frequency. As a role, the vibrating system is designed on the basis of a half-wave resonant assembly implying that lengths of the vibrating parts are equal to half-wave length.

Figure 1 illustrates a typical construction of ultrasonic module consisting of a magnetostrictive transducer, horn, sonotrode and radiator. By sonotrode is meant a confined elastic medium capable of transmitting vibrations from transducer to a material being processed (load). Horn, or velocity transformer, is a sonotrode whose cross section varies longitudinally according to a certain law. Horn serves for the transformation (in particular, amplification) of vibration amplitude and velocity, and for matching load with the transducer. Radiator is an element of vibrating system that is in direct contact with the load. Such a module can be used to supply acoustic energy to both the liquid and solid phases. Generally, application of magnetostrictive transducers to the gas phase sonoprocessing is quiet possible but, in this case, the electroacoustic efficiency is much less than that in the case of liquid and solid phases.

Sonic/ultrasonic modules for high temperature applications necessarily include a cooling system. Figure $\mathbf{2}$ is a schematical representation of ultrasonic module designed for sonoprocessing of molten metals. ${ }^{27)}$ The module consists of a magnetostrictive transducer connected to a waveguide-irradiator via a concentrator. All the parts are housed in a box filled with cooling water. Additionally, the inner

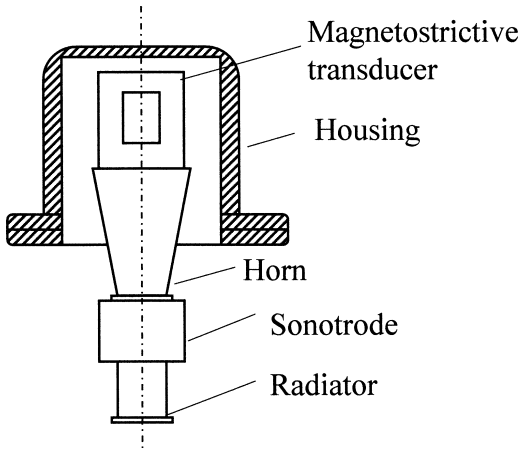

Fig. 1. A schematic representation of ultrasonic module.

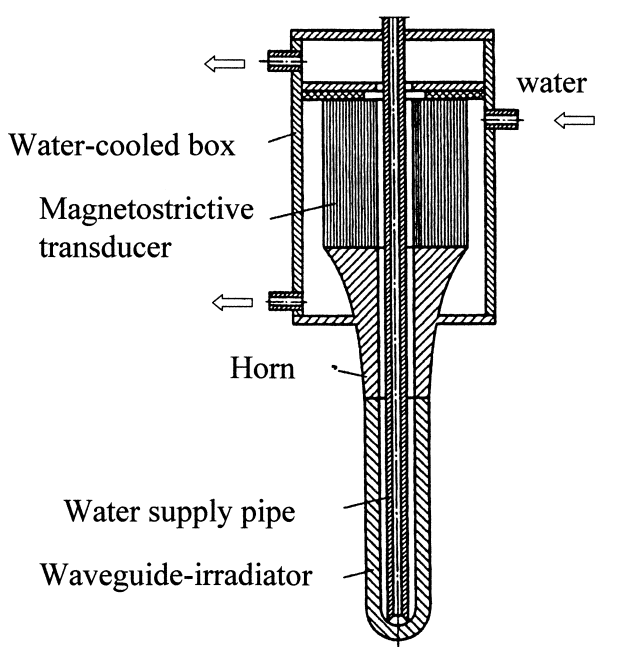

Fig. 2. A schematical representation of ultrasonic module for sonoprocessing of molten metals.

parts of the module are also cooled by passing water through a cooling tube at the module center. The transducer generates oscillations which, after amplifying by the concentrator, are transmitted to the waveguide-irradiator submerged into a molten liquid bath. This type of ultrasonic module has been tested for application to continuous casting of $\mathrm{Zn}, \mathrm{Al}$ and steel at a frequency of about $20 \mathrm{kHz}$.

\subsection{Mechanoacoustic Systems}

Mechanoacoustic systems are designed on the basis of transducers which convert mechanical energy into energy of acoustic oscillations. ${ }^{4)}$ They include pneumatic generators and special mechanical oscillators among which the former is more attractive for high temperature applications. The pneumatic generators use energy of compressed gas to produce a sound wave at collision of a gas stream with an obstacle. They are intended for gas-phase sonoprocessing, i.e. generated acoustic oscillations propagate through a gas phase. Depending on the principle of sound generation, pneumatic generators can be subdivided into two groups: sirens, in which gas flow is interrupted by a rotating element, and gas-jet generators (whistles), in which gas passes through a nozzle-resonator system. Although the mechanoacoustic efficiency of sirens can be as high as 50-60\%, they did not find wide practical application to high temperature processes. This is connected with a short durability of rotating parts of the sirens under the conditions of high temperatures and corrosive environments. The Hartmann- 


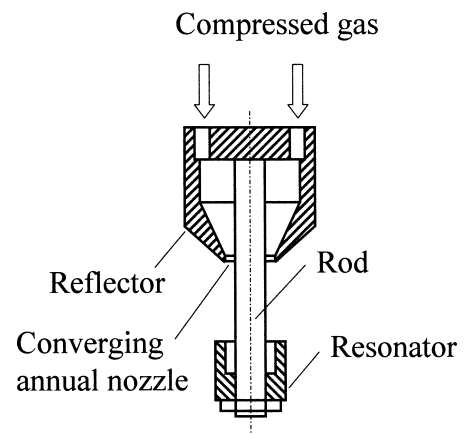

Fig. 3. A schematic representation of rod-type Hartmann generator.

type gas-jet whistles are the most promising transducer for pyrometallurgical applications.

There are several types of Hartmann whistles among which the rod-type Hartmann generator offers the most reliable performance. ${ }^{28)}$ Figure 3 shows a sketch of the rodtype generator. The main parts of the generator are a rod, converging annual nozzle, resonator and reflector. Although the principle of sound generation by the Hartmann whistles is still understood incompletely, one of the explanations of how the generator operates is as follows. An annual jet of compressed working gas issues through the nozzle at a supersonic velocity that results in perturbations of the jet structure and generation of waves of compression and rarefaction. These primary waves enter the resonator cavity and reflects from its bottom. It is thought that the interaction between the primary and reflected waves becomes a source of high-intensity acoustic oscillations.

Acoustic power of the rod-type Hartmann generator increases with the working gas flow rate which, in turn, is dependent on the thickness of annual slit of the nozzle. The sound frequency is governed by the distance between the nozzle edge and resonator bottom, and by some other geometric parameters. Generally, the generator can produce sound oscillations on frequencies from 0.5 to $40 \mathrm{kHz}$, at acoustic power up to a few $\mathrm{kW}$.

Obvious advantages of this type of sonic generator are compactness and simplicity in design. Another advantage is low cost of acoustic energy. Moreover, when the generator is applied to a process which uses a gas blowing or injection, the oscillations can be generated without any additional energy supply. One solution consists in building a generator into the blowing unit and passing a part of gas, used in the process, through the generator nozzle. Such a solution has been used to apply sound oscillations to steelmaking converter process. Finally, one more advantage of the Hartmann generators is simplicity of cooling the generator parts. In most cases, working gas provides a large enough heat sink from the generator parts to prevent them from overheating even when the generator is placed into high temperature environments.

\section{Sonoprocessing via Gas Phase}

\subsection{Acoustically Assisted Dedusting of High Tempera- ture Exhaust Gas}

Application of sonic oscillations for enlargement of fine particles suspended in a gas, has come to be known as acoustic agglomeration or coagulation. The first experiments on aggregating aerosol particles with the help of sound waves were conducted in the thirtieth years of the last century. ${ }^{29,30)}$ They initiated extensive studies of acoustic coagulation in USA, UK, Germany, Japan and Soviet Union. These studies were aimed at precipitating airborne liquid and solid particles at such processes as fossil fuel combustion, production of acids, soda ash and cement. It was found that sound waves promote the agglomeration of finer particles of submicron and micron size better than that of coarser particles. That is why the acoustic coagulation systems worked more effectively when they were applied together with cyclones, scrubbers, electro-static precipitators, etc., which are more effective for trapping coarser particles.

The first results on application of power sonic oscillations to pyrometallurgical processes were reported in the fifties of the last century in USA. ${ }^{31,32)}$ It was shown that dedusting efficiency of an open-hearth furnace flue gas can be significantly improved if the gas, before going to centrifugal-type dust separators, has been exposed to power sonic oscillations within a frequency range of $0-2.2 \mathrm{kHz}$. In these experiments, a half of dust particles had size less than $5 \mu \mathrm{m}$. An especially high degee of dust removal, up to $90 \%$, was discovered on injecting a small amount of water into the sonoprocessing chamber.

In parallel with experimental investigation, many theoretical works have been conducted concerning the mechanism of acoutic coagulation. Afterward, the results of all the above studies were summarized in an excellent book of Mednikov. ${ }^{8)}$

In succeeding years, many new studies were carried out with the use of more advanced instruments. ${ }^{33-35)}$ The obtained results allowed researchers to fill a lack of knowledge about key parameters influencing the acoustic coagulation. On the whole, the results confirmed the most of the earlier stated hypotheses on the acoustic coagulation mechanism.

The main feature of the all above-mentioned studies was that sonic oscillations were applied to process readyformed particles. Based on the results of these studies, key parameters influencing the acoustic coagulation can be summarized as follows. The coagulation rate increases proportionally to the square root of sound wave intensity and directly with the exposition time. Also, the coagulation rate increases with the particle number density in gas. The effect of frequency manifests itself mainly in two different ways depending on the process characteristics. 1) For any ensemble of polydisperse particles, there is the optimal frequency at which the coagulation rate is maximal. 2) Lower frequencies are always more efficient than higher frequencies.

A new approach to application of sound waves for pyrometallurgy was proposed in the former Soviet Union in the seventieth of the past century. The idea was that efficiency of sound waves can be appreciably increased when the waves are applied to gas phase inside the furnaces. In pyrometallurgical processes, the following four factors make the acoustic coagulation more effective when it is promoted inside the furnaces. 1) very high content of dust particles in gas atmosphere inside metallurgical units; 2) 
dust particles vary over a wide range in size; 3) high temperature of the dust particle surface. 4) when the sonic generator is installed inside a furnace, the noise can be significantly suppressed by a thick layer of the furnace lining.

The first attempts to realize this idea in practice were made by Blinov who designed several types of pneumatic sonic generators ${ }^{36-38)}$ and tested them on steelmaking converters. The main results of the tests have been reported here. ${ }^{9,40)}$ In one of the proposed methods, water-cooled pneumatic sonic generators of Hartmann type was built-in the tip of an oxygen lance between the lance nozzles. This type of lance will be called hereinafter acoustic lance. The main advantage of acoustic lances was that powerful sound waves of intensity about $1000 \mathrm{~W} / \mathrm{m}^{2}$ can be generated without any additional energy supply simply by passing a part of oxygen flow, used for metal refining, through the sonic generator nozzle. The working gas of generator, oxygen, after passing through the generator nozzle, merged with the main stream of oxygen discharged from the lance nozzles. Testing of such an acoustic lance was performed on both a pilot 10-t and industrial top-blowing 160-t converters within a wide range of frequencies from 2.2 to $13 \mathrm{kHz}$. The results revealed a significant reduction of dust content in flue gas due to application of acoustic lances.

Besides the acoustic lances, another type of acoustic stand-alone units was proposed. These units were also designed on the basis of Hartmann sonic generator, but differed from the acoustic lance in that the units can be installed regardless of oxygen lance position at any location inside the converter gas-collecting hood. ${ }^{41)}$ To generate sound waves, the acoustic units were supplied by compressed gas, air or nitrogen, which served also as a cooling gas for the unit parts. The acoustic units have a number of advantages over the acoustic lance. Firstly, they can be fixed at a safety distance from the molten bath. Secondly, few sonic generators can be installed at different locations in order to improve still further the acoustic coagulation efficiency.

The acoustic units have undergone numerous tests on industrial converters of various capacity. The resulst revealed that the acoustic units give the same effect as the acoustic lances. Figure 4 shows an example of time variation in total content of the dust in a flue gas during blowing in the 160 -t converter with use of a conventional five-nozzles oxygen lance (1), acoustic lance with the same number of nozzles (2) and combination of the conventional oxygen lance and acoustic unit (3). $\left.{ }^{9}\right)$ The unit was installed at $1.0-1.5 \mathrm{~m}$ above the converter mouth. In all cases, the dust samples were taken from the gas-collecting hood. As seen from Fig. 4 , the greatest difference in dust content between conditions with and without sonoprocessing was achieved during the second stage of blowing, from 5 to $12 \mathrm{~min}$. On average per heat, the total content of dust in flue gas was 56.5$59.4 \mathrm{~g} / \mathrm{m}^{3}$ when the conventional lance was used in these experiments. Application of sound resulted in a decrease in the total content by 38 and $35 \%$ with use of the acoustic lance and unit, respectively. These result relate mainly to the fine particles. As regards the coarse particles with diameter above $40 \mu \mathrm{m}$, their mass fraction in the dust was $10-15 \%$ without flue gas sonoprocessing and 5-7\% with it. Hence, sonic application reduced the content of coarse

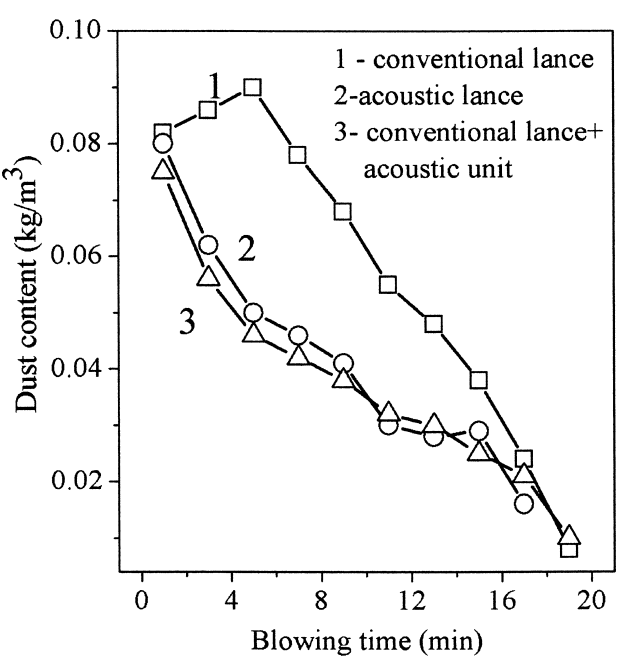

Fig. 4. Variation in dust content with blowing time.

particles to a greater extent than that of fine particles.

Along with the converter process, the same acoustic units have been tested for application to open-hearth furnaces. ${ }^{9,42)}$ Considering that the results can be of interest for researchers dealing with smelting processes in ferrous and non-ferrous metallurgy, they will be briefly indroduced below. The Hartmann generator based units were installed at the furnace bridge bank in such a way that sound waves propagate inside the furnace toward flow of exhaust gas leaving the furnace. The sound frequency and intensity varied within the limits of $2.5-3.0 \mathrm{kHz}$ and $3.0-6.0 \mathrm{~kW}$, respectively. Under these conditions, gas sonoprocessing yielded a reduction in total dust content by $25-30 \%$ and increase in particle average size by $5-7 \%$. In addition, the authors noted a reduction in fuel consumption gained as a result of improvement in the furnace heat efficiency.

It should be noted that the results of the above testings in application of sound waves to converters and open-hearth furnaces did not confirm the presence of optimal frequency regarding the acoustic coagulation efficiency. Lower frequencies were always more efficient than higher within the frequency range examined. The assumed reason is the following. When a sound wave propagates through the furnace exhaust gas with dust loading of several tenth of $g$ per $\mathrm{m}^{3}$, a higher frequency wave is attenuated faster as compared to a lower frequency one. ${ }^{15)}$ The attenuation nechanism is briefly discussed in the above sections. As a result, the intensity of acoustic field in the furnace at lower frequencies is always higher than that at higher frequencies. On the other hand, when the dust content in gas is much less, a few $\mathrm{g}$ per $\mathrm{m}^{3}$, some mechanism of attenuation, for example the scattering, can be neglected. Under these conditions, the experimental data revealed the presence of optimal frequency corresponding to the highest rate of acoustic coagulation.

A few words should be said about infuence of temperature on the acoustic coagulation of dust particles. Though special attention was not paid to this question in the literature, it is expected that temperature is one of the key parameters controlling acoustic coagulation. Firstly, a rise in temperature causes an increase in gas viscosity that should improve involving the dust particles in oscillatory motion. Secondly, higher temperatures are favourable for the formation of agglomerates of particles after their collision. An 
experimental verification showed that acoustically assisted coagulation of $\mathrm{Zn}$ particles, suspended in Ar, proceeds faster at temperatures above $\mathrm{Zn}$ melting point. ${ }^{43)}$

\subsection{Forced Acoustic Oscillations in Combustion and Environmental Processes}

Numerous studies have shown that high-intensity forced acoustic oscillations can enhance gas-phase mass and heat transfer, ${ }^{44-46)}$ improve turbulent mixing ${ }^{47,48)}$ and significantly increase the entrainment characteristics of gas jets. ${ }^{49,50)}$ These findings have motivated extensive investigations on application of the acoustic oscillations to improve the process performances in combustion, environmental and waste treatment technologies. The results of these investigations have strongly suggested that forced acoustics offers very attractive possibilities for designing high temperature processes with improved combustion efficiency and low pollutant emission. In pyrometallurgical field, these results would be of considerable interest for people dealing with such energy intensive industrial processes as metal refining, smelting reduction, dust recycling and waste treatment. A brief review of typical results presenting acoustic effects on the combustion and pollutant emission is as follows.

\subsubsection{Improvement in Fuel Combustion Efficiency}

A number of studies reported that the acoustic oscillations can enhance combustion rate of pulverized liquid/ solid fuels. The results of one of the first study on this issue $^{51)}$ showed that an imposition of air vibrations on a steady gas flow yields about $15 \%$ augmentation of a single fuel droplet burning rate independently on the vibration frequency. More recently, Blaszczyk ${ }^{52)}$ studied acoustically distributed single fuel droplet combustion under various frequencies. He concluded that about $14 \%$ increase in fuel combustion rate can be achieved at $120-300 \mathrm{~Hz}$ frequency range at the sound intensity of 100-115 dB.

The influence of acoustic field on the evaporation/combustion rate coefficients of a kerosene single fuel droplet was investigated experimentally under standing wave conditions. ${ }^{53)}$ The conclusion was that the rate coefficients increased by $2-3$ times when the droplet was fixed at a velocity antinode position of the wave at frequencies $<100 \mathrm{~Hz}$ and relatively low sound pressure levels of 100-110 dB.

The authors ${ }^{54)}$ studied effects of acoustic oscillations on the evaporation rate of methanol droplets (diam. 50$150 \mu \mathrm{m}$ ) at room temperatures and found that a $100 \%$ increase in their evaporation rate can be obtained only in the presence of an acoustic field at a SPL of $160 \mathrm{~dB}$. There was a weak tendency toward an increase of the effect with frequency ranging from 410 to $1240 \mathrm{~Hz}$. Most of the above data support the mechanism in which the obtained enhancement of liquid fuel combustion occurs due to a better mixing between the fuel vapor and oxidant. In all the above studies, powerful loudspeakers were applied for sound generation.

Practically the same effects of acoustics were found on the combustion of solid fuel particles. Yavuzkurt et al. ${ }^{55)}$ investigated the effect of an acoustic field on the combustion of coal particles in a flame burner by injecting the particles of 20-70 $\mu \mathrm{m}$ into the burning gas stream and by monitoring the light intensity emitted from the flame. Averaged values of light intensity were 2.5-3.5 times higher at SPL of $145-150 \mathrm{~dB}$ and frequency of $2000 \mathrm{~Hz}$ compared to those without sound application. Additionally, the authors performed a numerical simulation of combustion phenomena of $100-\mu \mathrm{m}$ coal particles, the results of which revealed 15.7 and 30.2 percent decreases in the char burn-out time at frequency of $2000 \mathrm{~Hz}$ and sound intensity levels of 160 and $170 \mathrm{~dB}$, respectively. ${ }^{56)}$ The main reason for the char burning enhancement is that the high-intensity acoustic field induces an oscillating slip velocity over the coal particles which augments the heat and mass transfer rates at the particle surface.

Koepke et al. ${ }^{57)}$ used four loudspeakers to apply an acoustic field to $125-\mu \mathrm{m}$ black liquor solid particles, injected into a reactor tube at a gas temperature of $550^{\circ} \mathrm{C}$. The intensity of the field was $151 \mathrm{~dB}$, frequency was ranged from 300 to $1000 \mathrm{~Hz}$. The results revealed a $10 \%$ reduction of char yield compared with that obtained without acoustic field application. Besides, significantly increased yields of product gases $\mathrm{CO}$ and $\mathrm{CO}_{2}$ were also observed with acoustic treatment. On the whole, the results revealed that the acoustic effects were more pronounced for the initial period of particle heat-up. The above two works ${ }^{55,57)}$ also include brief overviews of earlier publications on the acoustically improved fuel combustion.

In gas-fuelled combustion processes, the forced acoustic oscillations were found to be helpful in controlling flame shape and solving such problems as flame instability, lean flammability, reattachment to burners and poor combustion, especially when combustion occurs under diffusion flame conditions. Typical examples of diffusion flames are nonpremixed jet flames which are widely used in industry for safety reasons, and flames produced during post-combustion operations.

Especially great effect of acoustics was found at resonance frequencies, i.e. when the frequency of imposed oscillations was adjusted to the natural frequency of the combustor chamber. Yoshida et al. ${ }^{50)}$ studied behaviors of diffusion methane flame under an acoustic excitation at resonance frequencies of 900, 1350 and $2200 \mathrm{~Hz}$ and SPL of $112 \mathrm{~dB}$. Their results showed that at the resonance conditions, the turbulent intensity of the flow increases markedly, the flame becomes much wider and shorter than that without excitation. Similar acoustic effects were reported by Demare and Bailott ${ }^{58)}$ who applied acoustic oscillations to a methane/air nonpremixed flame at a non-resonant frequency of $200 \mathrm{~Hz}$ by using a powerful loudspeaker. Apparently, therefore, the authors had to increase SPL of acoustic oscillations up to $160 \mathrm{~dB}$ and higher. Hardalupas and Selbach ${ }^{59)}$ imposed oscillations on the coaxial air flow of a Swirl-stabilized burner with natural gas fuel injected axially at the flow center at frequencies of $200-920 \mathrm{~Hz}$ including a resonance one at $350 \mathrm{~Hz}$. In their experiments, the acoustic oscillations improved the lean-flammability limit by up to $15 \%$ with little frequency dependence. This work also provides a good review of earlier publications on controlling flame shape, stability and heat transfer.

\subsubsection{Reduction of Combustion-related Pollutant Emis- sion}

In parallel with the combustion enhancement, forced 
acoustic oscillations provide a way to significantly reduce emission of such pollutions as $\mathrm{NO}_{x}, \mathrm{CO}$ and soot particulates. Especially, a large body of literature has been published on suppressing the $\mathrm{NO}_{x}$ formation due to acoustically or mechanically imposed oscillations. Good reviews on this topic can be found in the relevant literature, for example. ${ }^{59-61)} \mathrm{NO}_{x}$ reduction level was found to be strongly dependent on the experimental conditions. The reported values are ranged from $100^{61)}$ to $15 \%{ }^{62)}$ decrease in $\mathrm{NO}_{x}$ emission rate as compared with that for steady flow conditions. The suppression mechanism has been well established. A sound wave, being propagated through a gas, can be thought as turbulent flow fluctuations of certain scale and amplitude which are governed by the wave frequency and intensity, respectively. Thus, imposing acoustic oscillations on flame front enhances the turbulent mixing resulting in reduced peak temperatures at the front that, in turn, is the reason of reduced emission of thermal $\mathrm{NO}_{x}$. When acoustic field is imposed upon flame containing liquid/solid particles, oscillations of gas around the particles provide an additional mechanism of the peak temperature reduction due to convection. One more reason of low $\mathrm{NO}_{x}$ emission is that high amplitude acoustic oscillations induce a strong recirculation of flue gas inside the combustion chamber. This results in entrainment of the already formed $\mathrm{NO}_{x}$ into the flame zone where $\mathrm{NO}_{x}$ is reduced by hydrocarbon radicals homogeneously or heterogeneously on the surface of carbonaceous solid particles. The same mechanism causes lowering of emission of $\mathrm{CO}$ and other gaseous pollutants although the literature on this subject is much less than that on the $\mathrm{NO}_{x}$ emission control.

For example, a large decrease in $\mathrm{NO}$ and $\mathrm{CO}$ emissions was observed in the presence of acoustic oscillations imposed to an ethanol flame in a Rujke tube pulse combustor. ${ }^{60)}$ Taking concentration values at steady conditions as a reference, the decreases were $52-100 \%$ for NO and 53$90 \%$ for CO depending on SPL (136 to $146 \mathrm{~dB}$ ), frequency $(80$ to $240 \mathrm{~Hz})$ and excess air $(10$ to $50 \%)$. Another example is the work $^{62)}$ the authors of which obtained emissions levels of a premixed methane-air flame below $5 \mathrm{ppm}$ for $\mathrm{NO}_{x}$ and $\mathrm{CO}$.

Few studies examined the effect of forced acoustics on soot emission from different types of flame: a spray ethanol flame of a Rijke tube combustor, ${ }^{60)}$ acetylene $^{63)}$ and methane diffusion flames. ${ }^{58,64)}$ The oscillation frequencies were also different: $200 \mathrm{~Hz},{ }^{58)} 40-240 \mathrm{~Hz},{ }^{60)}<100 \mathrm{~Hz}^{63)}$ and $40-1000 \mathrm{~Hz}^{64)}$ In spite of such different conditions, all the authors reported full disappearance of soot emission from the flames with acoustic excitation. The results of these studies suggested that acoustic oscillations enhance the mixing of fuel and ambient gas that causes a re-oxidation of soot particles at the flame zone.

\subsubsection{Improvement in Hazardous Waste Incineration}

Application of forced acoustics to waste incineration technology is a new and very attractive technique in sonoprocessing. This is presently being developed to solve a number of important problems in environment protection and capital cost saving. Most beneficial results of the recent publications are that acoustic oscillations provide possibilities to make the incinerator more compact and to achieve low pollutant emissions at once, especially under resonance frequency conditions.

Stewart et $a l .{ }^{65)}$ retrofitted a bench-scale rotary kiln incinerator simulator with a frequency-tunable acoustic pulse combustor and measured CO, total hydrocarbon (THC) and soot content in flue gas immediately downstream of the kiln. A toluene-containing substance was used as a matter modeling hazardous waste charge. The authors found that high amplitude acoustic oscillations has a strong tendency to reduce the amount of soot and/or semi-volatile and nonvolatile THC. Willis et al. ${ }^{66)}$ examined destruction of gaseous (sulfur hexafluoride) and liquid (acetonitrile) surrogates injected into recirculation zones within an acoustically excited propane-fuelled dump combustor. Although destruction efficiency was affected by the experimental variables in a complicated manner, the authors found conditions under which the surrogates, especially $\mathrm{SF}_{6}$, were destroyed well. In their experiments, these conditions were associated with recirculation zone and flame stability. Chun and Shin $^{67)}$ were able to achieve more than $99.99 \%$ DRE (destruction and removal efficiency) of hazardous waste and, simultaneously, to reduce NO emission by nearly $60 \%$ by applying acoustic oscillations to a $3.2 \mathrm{~kW}$ laboratory scale cavity-type incinerator. The study ${ }^{68)}$ focused on the enhancement of hazardous waste and pyrolysis gas surrogate destruction in an acoustically resonant dump combustor. The waste surrogate was injected into recirculation zones within the combustor. This allowed the surrogates to be trapped for relatively long residence time under potentially high temperature and/or relatively oxygen-rich conditions so that they could be destroyed more efficiently. Emissions of NO were found to decrease by nearly $60 \%$, unburned hydrocarbons dropped by over two orders of magnitude, and waste and pyrolysis gas surrogate destruction increased by nearly three orders of magnitude, all with external forcing at a resonant mode of $295 \mathrm{~Hz}$ by using a loudspeaker.

Few studies have provided interesting data that the forced acoustics can help in improving the performance of incinerated waste afterburners. Acoustically based active control of fluid dynamics has been used to enhance mixing in incinerator afterburner experiments in order to increase the DRE of a waste surrogate. ${ }^{69)}$ A gaseous fueled $4.5 \mathrm{~kW}$ incinerator was able to surpass $99.997 \%$ DRE even when the waste surrogate (gaseous benzene) constituted $66 \%$ of the total fuel content. The DRE for liquid afterburner/incinerator of $56 \mathrm{~kW}$ energy release exceeded $99.999 \%$ when combustible to air ratio was kept below 0.91 . The results of another study ${ }^{70)}$ showed that gaseous pollutants from the pyrolysis of realistic solid waste can be effectively burned out in an acoustically forced afterburner operated at a resonance frequency of $200 \mathrm{~Hz}$. The authors pointed out that in addition to very low pollutant emissions, the critical advantage of the acoustically forced afterburner is its compact size. The afterburner can achieve excellent burnout in a chamber volume that is 20 times smaller than conventional afterburner designs. 


\section{Ultrasonic Treatment of Melts}

\subsection{Ultrasonic Atomization of Liquid Metals}

Breaking liquid into fine droplets due to acoustic energy is known as ultrasonic atomization. The ultrasonic atomization of liquid metals is one of the promising application of high-intensity ultrasound to pyrometallurgy. There is a large body of literature on the physical principle and equipment design for ultrasonic atomization of liquids including melts, e.g. ${ }^{4,72}$ This section briefly introduces some earlier and recent studies on ultrasonic atomization of melts with emphasis on their potential application in practice.

Ultrasonic vibrations can be transmitted to atomization zone through melt or gas phases. Accordingly, ultrasonic atomization methods are subdivided into two groups ${ }^{72)}$ : a) ultrasonic vibratory atomization, b) ultrasonic gas atomization (USGA). In the first method, the melt passes as a film over a vibrating surface and droplets are formed directly from this film. Inevitable contact between the surface and melt restricts application of the vibratory atomization to melts with comparatively low melting points. Besides, this method has a low productivity. In the USGA method, the melt stream, flowing out of a vessel from an outlet nozzle, is exposed to high-intensity sonic or ultrasonic waves propagating to the melt through gas atmosphere. Most USGA methods use pneumatic sonic generators, for example those of Hartmann type, the operating principle of which was described in the previous sections. The USGA method is more attractive for practical use because of its high productivity and some other advantages over conventional methods.

Another variation of ultrasonic gas atomization exploits acoustic forces acting upon a liquid jet at a pressure node of a standing wave field. ${ }^{72,73)}$ The authors named the method ultrasonic standing wave atomization (USWA).

Pohlman and co-authors were among the first to investigate the ultrasonic vibratory atomization for producing powders from such low-melting point metals as $\mathrm{Pb}, \mathrm{Zn}, \mathrm{Cd}$, $\mathrm{Bi}^{75)}$ Atomization occurred under an atmosphere of inert gases and produced spherical powder with an extremely narrow size distribution under a frequency of $20 \mathrm{kHz}$. They emphasized a high efficiency of the proposed process. If energy requirement for production of 1 ton of lead powder by conventional ball mill method is $357 \mathrm{kWh}$ with an output rate of $8 \mathrm{~kg} / \mathrm{h}$, as little as $42 \mathrm{kWh}$ are required to produce the same amount of powder by the proposed ultrasonic equipment with an output rate of $45 \mathrm{~kg} / \mathrm{h}$.

Grant et al ${ }^{76-79)}$ developed an ultrasonic gas-jet atomizer for powder production of $\mathrm{Al}$ alloys and stainless steels. The size distribution of obtained powders was strongly depen- dent on gas pressure and extent of melt superheating. For an $\mathrm{Al}$ alloy, ${ }^{77)}$ the dendrite cell size grew linearly with particle dimensions up to a value of $100 \mu \mathrm{m}$, whereupon varied insignificantly. Based on these data, the authors were able to calculate the cooling rate, which was about $10^{5} \mathrm{C} / \mathrm{s}$ for particles smaller than $100 \mu \mathrm{m}$ in size. Additionally, Grant's group $^{80)}$ has demonstrated that a good yield of amorphous and microcrystalline $\mathrm{Cu}_{0.6} \mathrm{Zr}_{0.4}$ powder can be achieved by USGA. The differential weight distribution of produced particles peaked near $44 \mu \mathrm{m}$ with half particles being under $63 \mu \mathrm{m}$.

Clyne et al. ${ }^{81,82)}$ conducted an investigation on the solidification behavior of $\mathrm{Al}-\mathrm{Li}$ alloy droplets during ultrasonic gas atomization in a stream of He. The powders produced consisted of relatively fine particles with initial mass median diameter of about $30 \mu \mathrm{m}$ and of numerous very fine particles with diameters below 2 to $3 \mu \mathrm{m}$ which exhibited no cellular substructure or other evidence of chemical inhomogeneity.

Few metals with low melting temperature, namely Sn, $\mathrm{Sn}-\mathrm{Pb}$ and $\mathrm{Sn}-\mathrm{Bi}$ alloys, were used to examine the performance of the above mentioned USWA for metal powder production. ${ }^{72,73)}$ It was shown that spherical metal powders with minimum mass median diameters of less than $15 \mu \mathrm{m}$ can be generated at production rates of several $\mathrm{kg} / \mathrm{h}$.

Attempts to atomize molten steels by the ultrasonic gas atomization were undertaken by Abramov et al. ${ }^{83-85)} \mathrm{A}$ high-speed tool steel comprised $1 \% \mathrm{C}, 6 \% \mathrm{~W}, 5 \% \mathrm{Mo}$, $4 \% \mathrm{Cr}$ and $2 \% \mathrm{~V}$ was chosen for the investigations. An ultrasonic atomizer pneumatic operated at $14-15 \mathrm{kHz}$ and sound pressure level of $176-180 \mathrm{~dB}$. Pressure of nitrogen, which was served as a working gas, was varied from 1.0 to 1.2 $\mathrm{MPa}$. Parameters examined were pouring temperature, gas flow rate and diameter of molten steel stream. The asobtained powders were analyzed for size distribution and oxygen concentration. Additionally, the powders were compacted by hot extrusion at $1100-1150^{\circ} \mathrm{C}$ and, after annealing, the extruded blanks were subjected to quenching and triple tempering for $1 \mathrm{~h}$ at $560^{\circ} \mathrm{C}$ followed by testing for hardness, impact properties and bending strength.

The results revealed that ultrasonic oscillations improved the powder yield by $10-15 \%$, significantly increased the fraction of finer particles and made the particles size distribution more narrow as compared with the conventional pressure jet atomization. Table $\mathbf{2}$ compares size distributions of steel powders produced by the conventional atomization and USGA for different gas flow rates. Metallographic analysis confirmed the dendritic structure of particles surface and indicated an increasing structure refinement with diminishing particle size.

The most interesting result obtained in this study was

Table 2. Characteristics of powders produced by conventional atomization and USGA method.

\begin{tabular}{|l|l|l|l|l|l|l|l|l|}
\hline \multirow{2}{*}{ Atomization } & \multicolumn{5}{|c|}{ Fraction of particles within a given size } & \multirow{2}{*}{$\begin{array}{l}\text { Mean } \\
\text { size, } \mu \mathrm{m}\end{array}$} & $\begin{array}{l}\text { Gas } \\
\text { rate } \\
\end{array}$ \\
\cline { 2 - 9 } & $<50$ & $50-100$ & $100-160$ & $160-315$ & $315-500$ & $>500$ & & m $^{3} \mathrm{~kg}^{-1}$ \\
\hline Convention. & 4.6 & 29.8 & 16.7 & 12.2 & 13.0 & 23.7 & 122 & 0.67 \\
\hline Convention. & 9.5 & 30.5 & 20.0 & 12.5 & 10.0 & 15.7 & 107 & 1.03 \\
\hline USGA & 18.6 & 39.9 & 14.2 & 14.8 & 5.0 & 7.5 & 91 & 0.65 \\
\hline USGA & 27.2 & 52.7 & 7.8 & 6.3 & 0.0 & 6.0 & 81 & 0.90 \\
\hline
\end{tabular}


that the steel powder produced by USGA contained an order of magnitude less oxygen, $300 \mathrm{ppm}$ against $3000 \mathrm{ppm}$ in powder produced by conventional atomization technique. It is suggested that the lowered content of oxygen was responsible for improvements in the mechanical properties of ultrasonically atomized steel powders. Thus, impact property was increased from $2-3 \times 10^{5}$ to $3-5 \times 10^{5} \mathrm{~J} / \mathrm{m}^{2}$, bending strength from $2600-3600$ to $3400-5100 \mathrm{MPa}$. These improvements resulted in 1.2-1.4 times longer tooling life for tools made of ultrasonically produced powders as compared to conventional powders.

\subsection{Structural Modification of Solidifying Metals}

Starting from pioneering works of Sokolov, ${ }^{2,3)}$ a great body of experiments has been conducted concerning the effects of ultrasonic irradiation on structure and properties of as-cast metals and alloys. Pure metals $\mathrm{Zn}, \mathrm{Al}, \mathrm{Bi}, \mathrm{Cr}$, Ti, Mo, ${ }^{86-88)}$ light-metal alloys ${ }^{89)}$ copper alloys ${ }^{90)}$, irons and steels $^{91)}$ are notable examples of material systems investigated, although this list is far from complete. Results of these investigations revealed that stucture of solidifying metals is influenced by ultrasonic vibrations to a variable degree, which is termed the ultrasonic treatability. It is said that material possesses a good ultrasonic treatability if appreciable structural changes can be achieved using ultrasonic of a relatively low power, and vice versa. For example, among pure metals, Ti has the worst ultrasonic treatability.

In this section, we will introduce the effects of ultrasonic oscillations on structure of solidifying metals and present some results of pilot and industrial tests on ultrasonic treatment of $\mathrm{Al}$ and $\mathrm{Fe}$ alloys both of which are of great commercial interest.

In order to treat a solidifying metal with ultrasound, the energy of vibrations must be supplied directly to the solidifying interface. On the whole, there are two methods of supplying the ultrasonic energy to the solidifying front: 1through the molten bath, 2-through the solid which presents the solidified part of ingot.

Generally, effects of ultrasonic on solidifying metals can be summarized as follows.

1) Reduction in the mean grain size;

2) Suppression of columnar grain growth;

3) Variation in the distribution of phases in terms of their relative amounts, structural refinement, and mutual geometry;

4) Improvement in ingot homogeneity and segregation control;

5) Uniform distribution of nonmetallic inclusions.

At least, two phenomena are responsible for the above effects of ultrasonics. The first one is cavitation which was briefly considered in the previous section. Cavitation occurs with collapse of bubbles producing microjets. When cavitation tooks place close to solidifying interface, the microjets impact the crystal interfaces that can result in breaking of the crystals and in carrying of their fragments away from the interface. The second one is acoustic streaming occurring in the melt bulk. The streaming causes a vigorous mixing of the melt that promotes homogenization of the melt in chemical composition and temperature.

\subsubsection{Al Based Alloys}

Historically, Seeman and Menzel ${ }^{92)}$ were the first who applied ultrasonics to continuous casting of Al. They used a $25-\mathrm{kW}$ oscillator, operated at $40 \mathrm{kHz}$, for ultrasonic treatment of a round billel of $290 \mathrm{~mm}$ in diameter. Ultrasonic irradiation was found to refine both macro- and microstructure of ingots that improved their mechanical properties.

Extensive studies on the ultrasonically assisted solidification of $\mathrm{Al}$ alloys were undertaken by Eskin and coworkers $^{93-99)}$ in the former Soviet Union. Their experiments cover a wide range of systems including pure Al, model $\mathrm{Al}-\mathrm{Cu}$ alloys, $\mathrm{Al}-\mathrm{Mn}, \mathrm{Al}-\mathrm{Zr}$ and $\mathrm{Al}-\mathrm{Cr}$ alloys with intermetallic compounds, and commercial Al-based alloys as well. The general result for all the systems examined was that ultrasonic irradiation causes refinement of grains in the alloys after solidifying. However, the effect was found to be dependent on the alloy composition, temperature and some other factors. For example, in pure $\mathrm{Al}$, structural changes caused by ultrasonic were insignificant. On the other hand, in $\mathrm{Al}$ modified by a small amount of $\mathrm{Ti}, \mathrm{Zr}$ or boron carbide, ultrasonic irradiation resulted in reductions of marcograin and micrograin sizes by 40 and 10 times, respectively. In model $\mathrm{Al}-\mathrm{Cu}$ alloys, the efficiency of ultrasonic treatment on the grain refinement rose with increasing the copper concentration from 2 to $33 \%$.

In $\mathrm{Al}-\mathrm{Mn}$ alloys comprising 1 to 5 weight percent manganese, ultrasonic was found to reduce size of $\mathrm{Al}_{6} \mathrm{Mn}$ intermetallic particles. This resulted in a significant improvement in mechanical properties of the alloys raising tensile strength from 170 to $210 \mathrm{MPa}$, yield strength from 140 to $175 \mathrm{MPa}$, and elongation from 12.5 to $17.5 \%$.

Table 3 summarizes the effects of ultrasonics on the properties of some commercial Al alloys investigated by the group of G.I. Eskin. They found that, along with the structure refinement, ultrasonic can substantially suppress zonal segregation of $\mathrm{Zr}, \mathrm{Ti}, \mathrm{Cr}$, and $\mathrm{Mn}$ in the $\mathrm{Al}$ alloys.

Based on the results of the above studies, Eskin and coworkers $^{94)}$ determined the conditions of continuous casting, under which a dendrite-free grain structure was formed. This type of structure could be observed, if the grain size was smaller than or equal to the size of a dendrite cell produced at a given solidification rate. Cavitation in the melt appeared to be the necessary condition for an as-cast subdendrite structure to develop. As a result, they could produce $850-\mathrm{mm}$ diameter cast billets from most commercially available inoculated aluminum alloys with the subdendrite structure.

A number of studies was devoted to ultrasonic treatment of Al-Si alloys. Abramov ${ }^{100)}$ examined the joint effects of ultrasonic irradiation and inoculation on the structure of Al-14\%Si alloy. Sodium, as a microadditive, was introduced to the melt at concentration of $0.1 \%$ immediately before pouring. The macrostructure of untreated cast alloy showed the presence of the large segregates of excess $\mathrm{Si}$ crystals against the background of relatively coarse eutectics. By contrast, ultrasonic irradiation and microalloying with $\mathrm{Na}$ refined silicon crystals and uniformly distributed them over the ingot.

Japanese reseachers ${ }^{101-103)}$ reported that the effect of ultrasonic vibrations on crystal structure of Al-Si alloys de- 
Table 3. Effect of ultrasonics on the mechanical properties of commercial aluminum-based alloys.

\begin{tabular}{|c|c|c|c|c|c|c|c|}
\hline $\begin{array}{l}\text { Alloy } \\
\text { grade }\end{array}$ & $\begin{array}{l}\text { Alloy } \\
\text { system }\end{array}$ & $\begin{array}{c}\text { Alloy } \\
\text { condition }\end{array}$ & $\begin{array}{l}\text { Ultrasonic } \\
\text { treatment }\end{array}$ & $\begin{array}{c}\sigma_{\mathrm{B}} \\
\mathrm{MPa}\end{array}$ & $\begin{array}{l}\delta \\
\%\end{array}$ & $\begin{array}{l}\Psi, \\
\%\end{array}$ & $\begin{array}{l}\text { Brinell } \\
\text { hardness }\end{array}$ \\
\hline \multirow[t]{4}{*}{ AL40 } & $\mathrm{Al}-\mathrm{Si}^{-} \mathrm{Cu}^{-}$ & As-cast & No & 160 & 5 & $\cdot$ & 64 \\
\hline & $\mathrm{Mg}-\mathrm{Ni}-\mathrm{Fe}$ & $"$ & Yes & 210 & 6 & - & 75 \\
\hline & & Heat-treated & No & 250 & 3 & - & 89 \\
\hline & & $"$ & Yes & 330 & 5 & - & 96 \\
\hline \multirow[t]{2}{*}{ AL19 } & $\mathrm{Al}-\mathrm{Cu}-\mathrm{Mn}$ & As-cast & No & 270 & 3 & - & $\cdot$ \\
\hline & & $"$ & Yes & 350 & 4 & $\cdot$ & - \\
\hline \multirow[t]{2}{*}{ AL20 } & & $"$ & No & 190 & 5 & 6 & 72 \\
\hline & & $"$ & Yes & 210 & 11 & 12 & 76 \\
\hline
\end{tabular}

Table 4. Effect of ultrasonic treatment on mechanical properties of high alloy steels.

\begin{tabular}{|c|c|c|c|c|}
\hline Steel grade & State & $\sigma_{\mathrm{B}, \mathrm{MPa}^{*}}$ & $\delta, \% *$ & $\Psi, \% *$ \\
\hline \multicolumn{5}{|l|}{ Ferrite steels } \\
\hline $0.15 \% \mathrm{C}-25 \% \mathrm{Cr}-0.6 \% \mathrm{Ti}$ & As-cast & $330 / 460^{* * *}$ & $12 / 14$ & $15 / 18$ \\
\hline $0.2 \% \mathrm{C}-27 \% \mathrm{Cr}$ & As-cast & $450 / 530$ & $6 / 20$ & $6 / 44$ \\
\hline $0.2 \% \mathrm{C}-27 \% \mathrm{Cr}$ & After heat treatment & $480 / 550$ & $14 / 20$ & $29 / 58$ \\
\hline \multicolumn{5}{|l|}{ Austenite steels } \\
\hline $0.2 \% \mathrm{C}-25 \% \mathrm{Cr}-20 \% \mathrm{Ni}$ & As cast & $460 / 460$ & $25 / 32$ & $44 / 71$ \\
\hline $0.2 \% \mathrm{C}-20 \% \mathrm{Cr}-20 \% \mathrm{Ni}-2 \% \mathrm{Mo}$ & As cast & $490 / 550$ & $42 / 54$ & $40 / 68$ \\
\hline
\end{tabular}

pends on the alloy composition. When ultrasound was applied to a hypo-eutectic Al-6\%Si alloy, a substantial fragmentation of dendrite primary crystals was observed only at a higher temperature range, $883-973 \mathrm{~K}$. On the other hand, irradiation of ultrasound to a hyper-eutectic $\mathrm{Al}-14 \% \mathrm{Si}$ alloy resulted in the crystal refinement even in the semisolid temperatures, $850-883 \mathrm{~K}$. The authors concluded that an ultrasonically induced acoustic streaming plays an important role in carrying and distributing of primary crystal segments over the molten alloys that prompts a nucleation of crystals in the melt bulk.

\subsubsection{Steels}

Until the present time, a considerable number of studies have been published concerning the ultrasonic application to control the structure of various steels. One of the first results concerning the effects of ultrasonics on steel structure were reported about 50 years ago. ${ }^{104,105)}$ The authors applied ultrasonics to solidifying austenite corrosion resistant steels and found significant refinement of the steel structure which yielded substantial improvement in their mechanical properties.

Later on, a large body of experiments were carried out by Abramov and coworkers. These experiments cover a broad range of plain-carbon, low- and high-alloy steels produced either by continuous casting or by the ingot-making. The authors developed two approaches for ultrasonic treatment of solidifying metals. First approach implied that the structural changes can be achieved by irradiating ultrasonics, the power of which is large enough for cavitation to occur near the solidifying interface. This approach was verified mainly with experiments on ingot casting. Second approach was based on the idea that ultrasonic irradiation can enhance the rate of heat transfer in the molten metal that should result in corresponding changes of ingot structure. This idea was proven to be attractive for continuous casting.

In the ingot casting processes, ultrasonic vibrations were transmitted to a solidifying interface through holes at the bottom of ingot mould. Generally, it was found that the ultrasonic treatability of plain carbon and low-alloy steels becomes better with increasing carbon content. Thus, structure of plain carbon steels with $\mathrm{C}<0.4 \%$ remained unchanged even after treating them with ultrasonics at as high specific power as $2.5 \mathrm{~kW} / \mathrm{kg}$. Alternatively, ultrasonic treatment of plain carbon steels with $0.8-1.0 \% \mathrm{C}$ provided already a substantial grain refinement at a less power, 1.5$2.0 \mathrm{~kW} / \mathrm{kg}$. This resulted in an increase in its tensile strength, elongation, and area reduction by about 75,30 , and $60 \%$, respectively.

However, ultrasonic treatment of high alloy steels yielded improvements in the ingot structure and properties even at lower carbon content. Especially, substantial effect was obtained after treating high chromium steels with ferrite structure. Table 4 presents some results concerning ultrasonic treatment of high alloy steels. Besides, ultasonic treatment has been tested during solidification of tool and boron steels, and Ni-based superalloy. Readers interested in more data on the ultrasonic treatment of steel during ingot casting are referred to the published books ${ }^{4,27)}$.

It has been established for a long time that continuous casting of many alloy steels occurs with formation of a number of defects. Typical and most hard-removable defects of continuous casting (c.c.) ingots of alloy steels are the centreline segregation of solute elements and shrinkage 


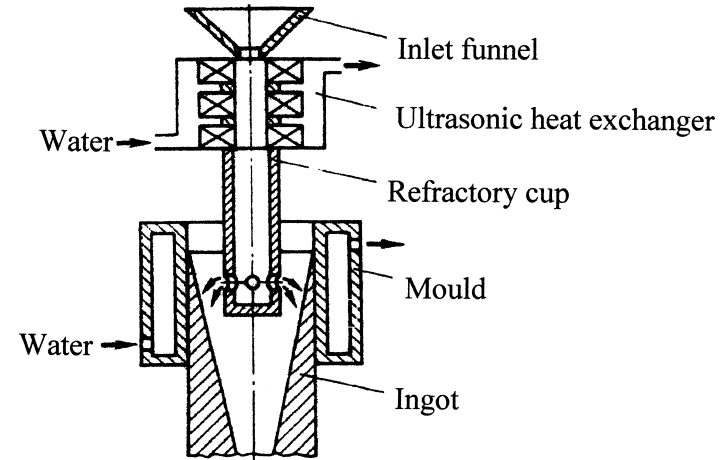

Fig. 5. A schematic representation of continuous casting through ultrasonic funnel.

cavities. These defects are mostly developed in alloy steels having a wide temperature range of solidification, such as high-carbon chromium bearing steels.

It was shown ${ }^{27,107)}$ that application of ultrasonic vibrations to solidifying steels in c.c. provides a means of suppressing both the carbon segregation and shrinkage cavities in a high-carbon chromium bearing steel. The following three units were designed and tested for ultrasonic treatment of the molten steel: a) water-cooled ultrasonic funnel, b) water-cooled ultrasonic irradiator,and c) vibrating consumable chiller.

Figure 5 is a schematical representation of the watercooled ultrasonic funnel. The flow of molten bearing steel was exposed to the ultrasonic vibrations at frequencies of $18-22 \mathrm{kHz}$ during passage through a copper tube mounted inside a water-cooled ultrasonic heat exchanger. A typical example of the water-cooled ultrasonic irradiator was shown in Fig. 2. The irradiator and chiller, although the latter is not shown here, presented ultrasonic waveguides which are immersed into the liquid pool. Thus, they can transmit the energy of ultrasonic vibations directly to the melt. The ultrasonic vibrations, regardless of waveguide type applied, intensified the heat exchange rate between the molten steel and the tube or mould, presumably due to a vigorous mixing of the melt. This resulted in a homogenization of the melt temperature and a reduction of the average temperature of steel in the mould by several tenth degrees as compared to that without ultrasonics. Lowering the temperature, in turn, led to an earlier formation equiaxed crystals during solidification that helped to lower the centreline carbon segregation and shrinkage cavity. Thus, ultrasonic irrdiation had an indirect influence on solidification process through controlling the heat transport phenomena.

The above units were tested during continious casting of bearing steels on a pilot and industrial scale equipment at steelmaking plants in Russia and Ukraine. The results can be summarized as follows.

1) During casting of the bearing steel (C-1\%,Cr-1.5\%) in a mould of $0.25 \times 0.36 \mathrm{~m}$ cross-section, ultrasonic vibrations provided increasing in the portion of ingot cross-sectional area occupied by the equiaxed crystals from 32 to $60 \%$ at an ultrasonic power of $1.2 \mathrm{~kW}$ and ingot drawing speed of $0.3-0.6 \mathrm{~m} / \mathrm{min}$.

2) For the all steels examined, the ultrasonically assisted suppression in the columnar grain growth yielded a more homogeneous structure of ingot including a more

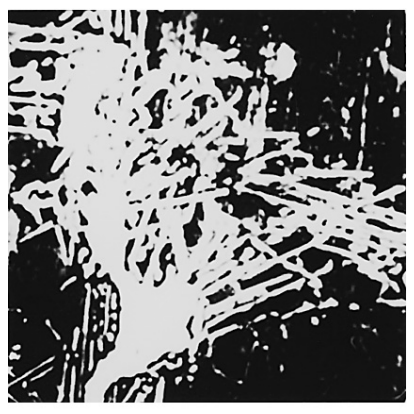

(a)

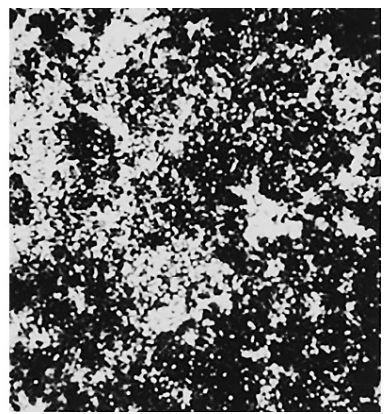

(b)
Fig. 6. Distribution of carbide inclusions over control (a) and ultrasonically treated (b) ingots of bearing steel grade comprising $1 \% \mathrm{C}$ and $1.5 \% \mathrm{Cr}$.

uniform distribution of carbides. An example of carbide distribution is shown in Fig. 6.

3) Structural changes in the ingots resulted in the rise of mechanical properties of steel. The tensile strength at room temperature increased from 49 to $63 \mathrm{kgf} / \mathrm{mm}^{2}$ elongation-from 10 to $18 \%$, contraction of cross-section-from 15 to $28 \%$, and impact strength increased from 1.5 to $2.8 \mathrm{kgm} / \mathrm{cm}^{2}$

4) The contact-fatigue durability tests of bearing steel have shown that the ultrasonic treatment raises service properties of the steel: guaranteed durability (N90) increased from 27.65 to 31.11 million of cycles (by $12.5 \%$ ) and mean durability (N50) from 99.4 to 141.5 million of cycles (by $42.5 \%$ )

Besides, the developed technology was applied during continuous casting of low-carbon steel $(0.15-0.20 \% \mathrm{C}$ and $0.6-0.8 \% \mathrm{Mn}$ ) in a mould with cross-section of $0.3 \times 1.85 \mathrm{~m}$. The ultrasonic oscillations were introduced into the melt by using a steel consumable chiller, the tip of which was immersed into the central zone of the molten steel pool. The tests revealed that crystallisation of metal in the slab central zone proceeds at a two fold rate under ultrasonic irradiation. As a result, the region of equiaxed grains in the centre of the ingot increased from $18.9-23.3 \%$ to $36.3-57.1 \%$ that yielded a substantial improvement in the steel mechanical properties.

Generally, it was concluded that application of ultrasonics to the molten pool is a very attractive technique of controlling the structure of continuous casting. This is supported by the following facts. The technique in which relativaly simple equipment is used, has a good adaptibility to continuous casting technology, is significantly cheaper and less energy-consuming in comparison, for example, to electromagnetic stirring.

\subsection{Preparation of Cast Composites}

Ultrasound can help in producing cast composites from immiscible metals or materials of different nature, for example metal matrix reinforced by ceramic particles. In the early 1960s, Pogodin-Alekseev and coworkers published a number of papers ${ }^{108-110)}$ in which he demonstrated the possibilities for using acoustic energy to produce metal base composite materials. Since then, many studies have been performed on ultrasonically assisted processes of composite production for several metal and metal-ceramic systems. 
This section provides a brief overview of experimental results on $\mathrm{Cr}-\mathrm{Ni}$-base heat-resistant alloys and Al-base alloys, and introduces a new ultrasonic technique for designing of composite materials.

The ultrasonic-base process of composite manufacture exploits the ability of power ultrasonics to generate macroand microstreaming due to the cavitation and acoustic streaming. The process can be subdivided into 3 stages.1melting of metal served as a matrix of composite, 2-distribution of solid particles or liquid droplets of the second phase over the liquid metal under ultrasonic irradiation, 3solidification of the metal matrix with or without ultrasonic irradiation.

Abramov and cooworkes ${ }^{111)}$ investigated behavour of various solid particles in a number of liquids subjected to ultrasonic irradiation under room temperatures. The results can be summarized as follows.

1) Time required for solid particles to be uniformly distributed over liquid bath increases as particle content, liquid viscosity and bath size increase, and as particle size and oscillation amplitude decreases. The particleto-liquid density ratio and wettability have less effects on the time.

2) In order for particles to be captured well by growing crystals, crystallization front velocity must exceed a critical value which, in turn, is dependent on particle size, wettability, density and thermoconductivity.

3) Cavitation promotes the particle capture and improves the performance of process under all conditions examined.

\subsubsection{Cr-Ni-base Heat-resistant Alloys}

Reinforcing heat-resistant metals and alloys by uniformly distributed ceramic particles in the metal matrix is a promising way to enhance the high-temperature strength of alloys. Such dispersion-hardened composite metals offer a number of properties which are unattainable in conventional heat-resistant metals. However, a direct inoculation of fine-grinded ceramic particles into a molten metal presents considerable difficulties, mainly because the wettability of many ceramics materials by liquid metals is poor.

$\mathrm{Al}_{2} \mathrm{O}_{3}, \mathrm{CeO}_{2}$ or $\mathrm{ZrO}_{2}$ particles of about $100 \mathrm{~nm}$ in size were used in an amount of $2 \mathrm{vol} \%$ to reinforce a Ni80Cr20 alloy matrix subjected to vacuum arc remelting. ${ }^{112)}$ To improve the oxide phase distribution, ultrasonic vibrations were applied to solidifying ingot. Microhardness measurements indicated an increase in the hardness of alloys from $210 \mathrm{HV}$ for the non-reinforced one to 220,220 and $256 \mathrm{HV}$ for alloys reinforced by $\mathrm{Al}_{2} \mathrm{O}_{3}, \mathrm{CeO}_{2}$ or $\mathrm{ZrO}_{2}$ particles, respectively. Thereupon, the authors measured characteristics of other two alloys, CrNi60Co and CrNi56VmoCoAl reinforced with $\mathrm{ZrO}_{2}$ particles in the same way.

All the dispersion-hardened alloys showed a higher structural stability in comparison with non-reinforced ingots. By the structural stability is meant here the tendency of materials to recrystallization. For example, the temperature of the recrystallization initiation, determined metallographically or from changes in the material microhardness after $1 \mathrm{~h}$ annealing, increased from $600^{\circ} \mathrm{C}$ for conventional Ni80Cr20 alloy to $1100-1200^{\circ} \mathrm{C}$ for alloy reinforced with $\mathrm{ZrO}_{2}$ particles.
It is especially noteworthy that ultrasonically assisted reinforcing method yielded more appreciate improvements in mechanical properties after long-term tests than those after short-term tests. For example, stress-rupture strengths of $\mathrm{ZrO}_{2}$-reinforced Ni80Cr20 alloy and non-reinforced were about the same after holding for $100 \mathrm{~h}$ at $1000^{\circ} \mathrm{C}$. Extension of holding time to $1000 \mathrm{~h}$ led to a large difference in the strengths: $15 \mathrm{MPa}$ for the reinforced alloy against $6 \mathrm{MPa}$ for the non-reinforced one. Furthemore, structural stability and stress-rupture strength, dispersionhardened alloys showed a better high-temperature compression resistance and higher oxidation resistance compared with conventional alloys.

\subsubsection{Al-base Alloys}

Other materials of great commercial interest are Al-base composites. Recently, several reports have demonstrated an exceptionally high effectiveness of ultrasonics in manufacturing some Al-base composite materials.

One group of them is $\mathrm{Al}-\mathrm{Pb}$ base composites which have a number of attractive properties, particularly, a high wear resistance. A uniform distribution of $\mathrm{Pb}$-rich phases over the height of ingots of $\mathrm{Al}-10 \% \mathrm{~Pb}-1 \% \mathrm{X}^{114)}$ or $\mathrm{Al}-10 \% \mathrm{~Pb}-$ $\left.10 \% \mathrm{Sn}-(0.5 \sim 6) \% \mathrm{X}^{115}\right)(\mathrm{X}=\mathrm{Si}, \mathrm{Cu}, \mathrm{Mg}, \mathrm{Sb})$ alloys was obtained during casting by applying ultrasonic vibrations alone, or in combination with electromagnetic forces. Analysis of the materials produced revealed that the $\mathrm{Pb}$-rich phases present in the Al matrix as finely dispersed inclusions with the size of $5-40 \mu \mathrm{m}$. All the Al-Pb base composites showed much higher wear and fatigue resistances as compared with those of tin-based babbits which are still in common use today as bearing materials. The conditions and results of other tests of the produced composites can be found in the above mentioned articles or in the published books. ${ }^{4,27)}$ More recently, Chen and Shu ${ }^{116)}$ applied high-intensity ultrasonics to prepare $\mathrm{Al}-\mathrm{Pb}$ composites and reported some details on the mechanism of homogeneous dispersion of the lead droplets in the Al matrix.

Among other composite materials those have received recent attention in connection with ultrasound application, are Al-base alloys reinforced by ceramic particles. Japanese researchers ${ }^{117)}$ investigated influences of particle shapes and sizes on the incorporation of $\mathrm{Al}_{2} \mathrm{O}_{3}$ particles into molten $\mathrm{Al}-5 \% \mathrm{Mg}$ alloy by melt stirring with ultrasonic vibration. Their results suggested that ultrasonic vibrations cause a significant reduction in the apparent contact angle between the particles and melt. Eskin ${ }^{118)}$ demonstrated that ultrasonic treatment combined with electromagnetic stirring can provide an uniformed distribution of 5-7 $\mu \mathrm{m} \mathrm{SiC} \mathrm{particles}$ in a $\mathrm{Al}-0.4 \% \mathrm{Si}-0.7 \% \mathrm{Mg}-20 \% \mathrm{SiC}$ composite. They also concluded that cavitation promotes the wetting of ceramic particles by liquid aluminum and involves them into solidification process. Yang et al. ${ }^{119)}$ proposed a new ultrasonic method for an inexpensive fabrication of a $\mathrm{Al}-7 \% \mathrm{Si}$ alloy based composite with reproducible microstructure and superior properties and achieved a uniform dispersion of nano-sized SiC particles in the matrix. The authors pointed out that the yield strength of the produced material could be improved more than $50 \%$ with only $2.0 \mathrm{wt} \%$ of nano-sized $\mathrm{SiC}$ particles as compared with non-reinforced alloy of the same composition. 


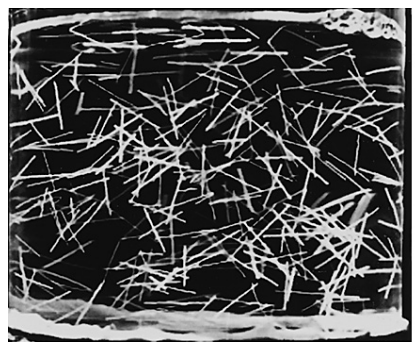

(a)

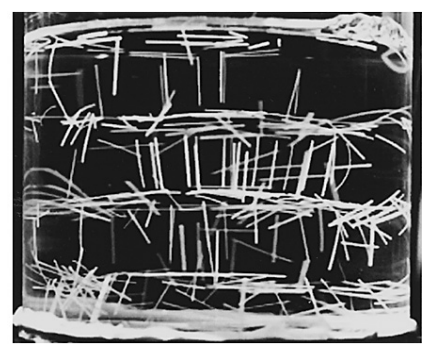

(b)
Fig. 7. Behavior of polystyrene fibers in aqueous sugar solution (a) without irradiation and (b) with irradiation of ultrasound at $46 \mathrm{kHz}$ under the conditions of $60 \mathrm{~mm}$ liquid depth and $5 \mathrm{~mm}$ fiber length.

\subsubsection{Ultrasonically Assisted Designing of Composite Materials}

As mentioned in the previous sections, ultrasonically induced radiation force provides a new option for controlling the distribution and orientation of particulates suspended in a liquid bath. This appears to be particularly promising for designing the structure of as-cast composite materials.

Cold model experiments ${ }^{74)}$ revealed that rod-like fibers shorter than one-fourth of the wavelength are constrained at the pressure node and are oriented in the direction perpendicular to that of wave propagation. On the other hand, fibers ranging from one-fourth to one-half of the wavelength were oriented either parallel to the direction of wave propagation at the pressure loop or perpendicular to that at the pressure node depending on their initial positions and directions. Figure 7 shows an example of the polystyrene fiber orientation in an aqueous sugar solution before ultrasound irradiation (a) and during irradiation (b) for $5 \mathrm{~s}$. The ultrasonic transducer was fixed at the vessel bottom. The experiments were carried out at a resonance standing wave at a frequency of $46 \mathrm{kHz}$.

Results of another cold model study ${ }^{106)}$ showed that when a resonance standing ultrasound wave is applied to a liquid containing randomly distributed spherical polystyrene particles, the radiation pressure forces them to gather at the pressure nodes or antinodes depending on the densities and acoustic properties of the liquid and particles. As a result, the particles forms a layered structure in the liquid with a distance between layers of half-wavelength. Thus, by varying ultrasonic frequency, one can design a composite material with a preference distance between layers. Moreover, the ultrasonic effect can be gained at a relatively low energy of acoustic filed if the liquid bath depth is adjusted so that a resonance condition can be achieved.

\section{Other Ultrasonic Applications}

There are at least two ultrasonic applications which remained beyond the scope of the present review, although they are potentially promising for pyrometallurgical application. The first one is the ultrasound degassing of liquid metals. The technique involves irradiating ultrasound into a molten bath at a large enough power to generate cavitation bubbles. The bubbles are assumed to be the main reason of the degassing effect. There is a great body of literature de- voted to promoting degassing of melts with ultrasonics, however almost all the studies concern alumium-based alloys, e.g. ${ }^{4,93,94)}$ The second one is the acoustically controlled slag foaming that consists in exposing the slag surface to a sound wave propagated to the slag through a gas phase. Although the method has been examined on laboratory scale equipment, ${ }^{39,71)}$ more data must be collected to confirm its adaptability for application to real industrial slags.

\section{Concluding Remarks}

Recently, considerable research efforts have been devoted to the investigation of poweful sonic and ultrasonic oscillations as a tool for improving the performance of metallurgical and other high temperature processes. The results of these investigations have strongly suggested that ultrasonics has the potential to play a more significant role in pyrometallurgy and related areas. At higher temperatures, attractiveness of sonic and ultrasonic waves is associated with its ability to propagate through fluids, gas or liquid, and thus to transfer the acoustic energy from a gas or water cooled ultrasonic generator to a higher temperature area for material processing. Furthemore, if the wave intensity is high enough, its propagation initiates such non-linear phenomena as cavitation, acoustic streaming and radiation pressure which are the prime causes of many ultrasonic effects in the fluid bulk and interface regions. These phenomena play an especially important role at high temperature interfaces because they provide an unique tool in controlling the interfacial mass and heat transfer, crystal growth, wetting and emulsification that can not be achieved by any other methods.

Industrial competitiveness of the ultrasonic-based technologies is reinforced by relatively low cost of power-generating equipment and ultrasonic transducers. In some special cases, the acoustic energy can be produced without any additional energy consumption by means of a very simple device. An example is the pneumatic sonic generator applied to a process which uses gas blowing or injection. However, by far the most complicated technical task in pyrometallurgical applications is designing effective and reliable waveguide systems capable of transferring acoustic energy from transducers into high temperature and chemically aggressive environments. Correspondingly, the waveguide systems are often the most costly parts of high temperature ultrasonic installations. Nevertheless, a number of comparatively simple solutions have been offered in this area, too. Typical examples include,first, transferring the acoustic energy through exhaust gas in the gas phase sonoprocessing and, second, through the solidified part of ingot. Further research work, however, is required in many areas, for example, regarding the development of new heat-resistant waveguide materials and the integration of ultrasonic installations with existing industrial facilities in high temperature technologies.

\section{Acknowledgements}

We would like to thank Prof. Hideaki Suito and Prof. Shoji Taniguchi (Tohoku University) for their comments and helpful discussion. 


\section{REFERENCES}

1) R. W. Wood and A. L. Loomis: Philos. Mag., 4 (1927), 417.

2) S. J. Sokolov: Acta Physicochim.USSR, 3 (1935), 939.

3) S. J. Sokolov: Tech. Phys. USSR, 3 (1936), 176.

4) O. V. Abramov: High-Intensity Ultrasonics: Theory and Industrial Applications, Gordon and Breach Science Publishers, Amsterdam, (1998), 692.

5) O. V. Abramov, S. G. Khorbenko and S. Shvelga: Ultrasonic Processing of Materials. Mashinostroenie, Moscow, (1984) [in Russian].

6) W. P. Mason and R. N. Thurston: Physical Acoustics, Vol. 4, Part B, Academic Press Inc., New York, (1965), 265.

7) M. B. Gitis and I. G. Mikhailov: Acoustic J., No. 12, (1965), 145.

8) E. P. Mednikov: Acoustic Coagulation and Precipitation of Aerosols, authorized translation from the Russian by Chas V. Larrick, USSR Academy of Sciences Press, Moscow, (1965), 180.

9) K. A. Blinov: Application of Acoustic Oscillations for Steelmaking Processes, Metallurgia, Chelyabinsk, Russia, (1991), 191 [in Russian].

10) L. D. Landau and E. M. Lifshits: Fluid Mechanics, Nauka, Moscow, (1986), 736

11) S. Temkin: J. Acoust. Soc. Am., 103 (1998), No. 2, 838.

12) J. Carlson and P. E. Martinsson: Ultrasonics, 39 (2002), 585.

13) M. F. Hamilton and D. T. Blackstock: Nonlinear Acoustics, Theory and Applications, Academic Press, California, (1998), 455.

14) J. W. S. Rayleigh: Theory of Sound, Dover Publications, New York, (1945), Vol. 1, 480, Vol. 2, 504.

15) M. J. Lighthill: Waves in Fluids, Cambridge University Press, Cambridge, (1978), 504

16) J. David and N. Cheeke: Boca Raton Fundamentals and Applications of Ultrasonic Waves, CRC Press, Boca Raton, (2003), 480.

17) S. Makarov and M. Ochmann: Acustica, 82 (1996), 579.

18) S. Makarov and M. Ochmann : Acustica, 83 (1997), 197.

19) T. G. Leighton: Int. J. Modern Phys. B., 18 (2004), 3267.

20) E. N. Harvey, D. K. Barnes, W. D. McElroy, A. H. Whiteley, D. C. Pease and K. W. Cooper: J. Cell. Comp. Physiol., 24 (1944), 17.

21) V. I. Dobatkin and G. I. Eskin, Effect of High-Power Ultrasound on Metal Interface, Nauka, Moscow, (1986), 6.

22) V. A. Akulichev: Intense Ultrasonic Fields, ed. by L. D. Goldberg, Nauka, Moscow, (1968), 129.

23) L. Crum: Ultrasonics, 22 (1984), 215

24) B. Flint and K.Suslick: J. Am. Chem. Soc., 111 (1989), 6987.

25) Z. A. Goldberg: Intense Ultrasonic Fields, ed. by L. D. Goldberg, Nauka, Moscow, (1968), 51.

26) Ultrasonic Transducers, ed. by Y. Kikuchi, Corona Pub. Co., Tokyo, (1969), 406

27) O. V. Abramov: Effects of High-Intensity Ultrasonics on Liquid and Solid Metals, Nauka, Moscow, (2000), 312 [in Russian].

28) Yu. Ya. Borisov: Sources of High-Intensity Ultrasound, Vol. 1, ed. by L. D. Rozenberg, Plenum Press, New York, (1969), 3-162.

29) H. S. Patterson and W. Cawood: Nature, 127 (1931), 667.

30) E. N. da C. Andrade: Philos. Trans. R. Soc. (London) A., 230A (1932), No. 692, 413.

31) C. R. Soderberg: Iron and Steel Eng., 29 (1952), No. 2, 87.

32) S. Vajda: Iron and Steel Eng., 29 (1952), No. 7, 111.

33) E. Riera-Franco de Sarabia and J. A. Gallego-Juarez: J. Sound Vibration, 110 (1986), 413.

34) J. Magill, Ph. Caperan, J. Somers and K. Richter: J. Aerosol Sci., 22 (1991), Suppl. 1, S27

35) T. L. Hoffmann and G. H. Koopmann: J. Acoust. Soc. Am., 99 (1996), No. 4, 2130

36) S. G. Afanasiev, K. A. Blinov, Yu. Ya. Borisov, Ya. D. Verbitsky, O. B. Havroshkin and Yu. V. Filatov: Inventors Certificate, USSR, No. 410099, Bulleten 1, (1974).

37) K. A. Blinov, A. D. Chertov, Ya. D. Verbitsky, A. I. Manohin, V. A. Ostrinsky, S. V. Kolpakov, A. M. Pozhivanov, S. Z. Afonin and Z. A. Alyamov: Inventors Certificate, USSR, No. 539951, Bulleten 47, (1976).

38) K. A. Blinov, Yu. Ya. Borisov, V. Yu. Volkov, S. Z. Afonin, Yu. G. Savvateev, V. A. Ostrinsky, Yu. E. Gorbenko and Z. A. Alyamov: Inventors Certificate, USSR, No. 779401, Bulleten 42, (1980).
39) S. Komarov, M. Kuabara and M. Sano: ISIJ Int., 40 (2000), 431.

40) K. A. Blinov and S. V. Komarov: Proc. 2nd Int. Symp. on Metallurgical Processes for the Year 2000 and Beyond, TMS, Warrendale, PA, (1994), 413.

41) K. A. Blinov, V. Yu. Volkov, A. M. Pozhivanov: Inventors Certificate, USSR, No. 699021, Bulleten 43, (1979).

42) V. G. Tyulebaev, V. M. Levin and Yu. F. Tyulebaeva: Metallurg, No. 5, (1986), 24.

$43)$ S. Komarov, T. Yamamoto, T. Uda and M. Hirasawa: ISIJ Int., 44 (2004), 275.

44) P. D. Richardson: Appl. Mech. Rev., 20 (1967), No. 3, 201.

45) P. S. Larsen and J. W. Jensen: Int. J. Heat Mass Transfer, 21 (1978), 511.

46) M. Y. Ha and S. Yavuzkurt: Int. J. Heat Mass Transfer, 36 (1993), 2183

47) P. J. Vermeulen, V. Ramesh and W. K. Yu: J. Eng. Gas Turbines Power, 108 (1986), 479.

48) P. S. Krueger and A. Gharib: AIAA J., 43 (2005), 792.

$49)$ E. C. Mladin and D. A. Zumbrunnen: Int. J. Heat Mass Transfer, 40 (1997), 3305.

50) H. Yoshida, M. Koda, Y. Ooishi, K. Kobayashi and M. Saito: Int. J. Heat Fluid Flow, 22 (2001), 372.

51) S. Kumagai and H. Isoda: Fifth Symp. (International) on Combustion, Reinhold Publishing, New York, (1954) 129.

52) J. Blaszyk: Fuel, 70 (1991), 1023.

53) M. Saito, M. Sato and I. Suzuki: Fuel, 73 (1994), 349.

54) R. I. Sujith, G. A. Waldherr, J. I. Jagoda and B. T. Zinn: J. Propulsion Power, 16 (2000), 278.

55) S. Yavuzkurt, M. Y. Ha, G. Reethof, G. Koopmann and A. W. Scaroni: Trans. ASME, J. Energy Resour. Technol., 113 (1991), 286.

56) S. Yavuzkurt, M. Y. Ha, G. Koopmann and A. W. Scaroni: Trans, ASME, J. Energy Resour. Technol., 113 (1991), 277.

57) S. A. Koepke and J. Y. Zhu: Combustion Sci. Technol., 140 (1998), 315.

58) D. Demare and F. Baillot: Combustion Flame, 139 (2004), 312.

59) Y. Hardalupas and A. Selbach: Prog. Energy Combustion Sci., 28 (2002), 75.

60) M. Q. Mcquay, R. K. Dubey and W. A. Nazeer: Fuel, 77 (1998), 425.

61) O. Delabroy, F. Lacas, T. Poinsot, S. Candel, T. Hoffmann, J. Hermann, S. Gleis and D. Vortmeyer: Combustion Sci. Technol., 119 (1996), 397.

62) J. O. Keller, T. T. Bramlette, P. K. Barr and J. R. Alvarez: Combustion Flame, 99 (1994), 460.

63) M. Saito, M. Sato and A. Nishimura: Fuel, 77 (1998), 973.

64) J. R. Hertzberg: Combustion Flame, 109 (1997), 314

65) C. R. Stewart, P. M. Lemieux and B. T. Zinn: Combustion Sci. Technol., 94 (1993), 427.

66) J. W. Willis, C. Cadou, M. Mitchell, A. R. Karagozian and O. I. Smith: Combustion Flame, 99 (1994), 280.

67) Y. N. Chun and D. Y. Shin: Kor. J. Chem. Eng., 21 (2004), 811.

68) G. Pont, C. P. Cadou, A. R. Karagozian and O. I. Smith: Combustion Flame, 113 (1998), 249.

69) E. J. Gutmark, T. P. Parr, K. J. Wilson, K. H. Yu, R. A. Smith, D. M. Hansonparr and K. C. Schadow: Combustion Sci. Technol., 121 (1996), 333.

70) J. A. Cole, N. C. Widmer, W. R. Seeker, K. C. Schadow, T. P. Parr and K. J. Wilson: Chemosphere, 42 (2001), 765.

71) S. Komarov, M. Kuwabara and M. Sano: Ultrasonic Sonochem., 7 (2000), 193.

72) O. Andersen, P. Schreckenberg and K. Bauckhage: Int. J. Powder Metall., 31 (1995), 341.

73) O. Andersen, S. Hanmann and K. Bauckhage: Part. Part. Syst. Charact., 13 (1996), 217.

74) S. Yamahira, S. Hatanaka, M. Kuwabara and S. Asai: Jpn. J. Appl. Phys., 39 (2000), 3683.

75) R. Pohlman and E. G. Lierke: Proc. of 5th Int. Cong. on Acoustics, ICA, Liege, (1965), D35.

76) N. J. Grant: J. Met., 35 (1983), 20.

77) N. J. Grant: Rapid Solification Processing, Plenum Press, New York, (1978), 230.

78) V. Anaud, A. J. Kaufman and N. J. Grant: Proc. of 2nd Int. Conf. on Rapid Solidification, Claitor's Pub. Division, Baton Rouge, (1980), 
273.

79) P. K. Domalavage, N. J. Grant and Y. Gefen: Metall. Trans. A, 14A (1983), 1599

80) P. K. Domalavage, N. J. Grant and Y. Gefen: Metall. Trans. A, 14A (1983), 1599.

81) T. W. Clyne, R. A. Ricks and P. J. Goodhew: Int. J. Rapid Solid., 1 (1984-85), 59 .

82) T. W. Clyne, R. A. Ricks and P. J. Goodhew: Int. J. Rapid Solid., 1 (1984-85), 85.

83) O. V. Abramov, Yu. Ya. Borisov and R. A. Oganyan: Akust. Zh, 27 (1981), 801 [in Russian].

84) O. V.Abramov, R. A. Oganyan and T. S. Shishkhanov: Poroshk. Metall., 6 (1981), 6.

85) O. V. Abramov, V. L. Gershov and R. A. Oganyan: Metalloved. Term. Obrab. Met., 37 (1982).

86) I. G. Polotskii: Proc. Inst. of Ferr. Metallurgy Acad. Sci. USSR, Izd, AN USSR, Kiev, (1963), 91

87) V. I. Danilov and G. Kh. Chedzhemov: Problems of Physical Metallurgy and Physics of Metals (in Russian), Metallurgizdat, Moscow, (1955), 34.

88) G. Schmid and L. Ehret: Zs. Electrochem., 43 (1937), 269.

89) G. I. Eskin: Ultrasonic Treatment of Molten Aluminum (in Russian), Metallurgiya, Moscow, (1965), 234.

90) G. I. Pogodin-Alekseev: Ultrasound and Low Frequency Vibrations in Alloy Production (in Russian), NTO, Mashprom, (1961),74.

91) I. G. Povolotsky: Application of Ultrasonic Oscillations for Property Investigations, Quality Control and Treatment of Metals and Alloys, AN USSR, Kiev, (1960), 78 [in Russian].

92) H. Seeman and H. Menzel: Zs. Metall., 1 (1947), 318.

93) G. I. Eskin: Ultrasonic Treatment of Molten Aluminum, Metallurgiya, Moscow, (1965), [in Russian].

94) G. I. Eskin: Effect of High-intensity Ultrasound on Metal Interfaces, Nauka, Moscow, 6 (1986), [in Russian].

95) G. I. Eskin: Ultrasonic Treatment of Melts in Shaped and Continuous Casting of Light Alloys, Mashinostroenie, Moscow, (1975), 57 [in Russian].

96) G. I. Eskin: Adv. Sonochem., 3 (1993).

97) G. I. Eskin: Ultrasound Sonochem., 2 (1995), 137.

98) G. I. Eskin and I. N. Fridlyander: Metalloved. Term. Obrab. Met., 4 (1962), 320 .
99) G. I. Eskin: Izv. AN SSSR, Ser. Metallurgiya i Toplivo, 1 (1963), 118.

100) O . V. Abramov: Crystallization of Metals in Ultrasonic Field, Metallurgiya, Moscow, (1972), 256 [in Russian].

101) Y. Osawa, G. Arakane, S. Takamori, A. Sato and O. Ohashi: Foundry Eng., 71 (1999), 98.

102) Y. Osawa and A. Sato: Foundry Eng., 72 (2000), 734

103) Y. Osawa, S. Takamori, G. Arakane, O. Umezawa, A. Sato and O. Ohashi: Foundry Eng., 72 (2000), 187.

104) S. S.Hinchliff and J. W. Jones: Foundry Trade J., 1 (1955), No. 9, 251.

105) J. Jagaciak and J. W. Jones : Foundry Trade J., 1 (1956) No. 10, 595.

106) S. Hatanaka, T. Taki, M. Kuwabara, M. Sano and S. Asai: Jpn. J. Appl. Phys., 38 (1999), 3096.

107) O. Abramov, A. Izotov, Yu. Astashkin, G. Kryuchkova, A. Manokhin, M. Markov and V. Taran: Steel, No. 4, (1985), 13 [in Russian].

108) G. I. Pogodin-Alerkseev and V. V. Zaboleev-Zotov: Liteinoe proizvodstvo, No.7, (1958), 35 [in Russian].

109) G. I. Pogodin-Alerkseev: Ultrasound and Low-freqency Vibration in Alloy Production, Mashgiz, Moscow, (1961), 71 [in Russian].

110) G. I. Pogodin-Alerkseev: Application of Ultrasound in Machinebuilding, MD NTR, Moscow, (1963), 72 [Russain].

111) O. V. Abramov, S. A. Govovanenko and I. V. Abramov: Metally, No. 6, (1972), 227.

112) I. V. Abramov, O. V. Abramov, V. B. Kireev: Met. Sci. Heat Treat., No. 12, (1975), 31.

113) I. V. Abramov, O. V. Abramov, V. B. Kireev, S. B. Maslenkov: Fizika i khimiya obrabotki materialov, No. 1, (1979), 54 [Russian].

114) O. V. Abramov, V. O. Abramov, F. Sommer and D. Orlov: Mater. Lett., 23 (1995), 17.

115) V. O. Abramov, O. V. Abramov, F. Sommer and D. Orlov: Mater. Lett., 29 (1996), 67.

116) F. Chen and G. Shu: J. Mater. Sci. Lett., 17 (1998), 259.

117) Y. Genma, Y. Tsunekawa, M. Okumiya and N. Mohri: Mater. Trans., 38 (1997), 239.

118) G. I. Eskin and D. G. Eskin: Ultrasonics Sonochem., 10 (2003), 297.

119) Y. Yang, J. Lan and X. Li: Mater. Sci. Eng., A 380 (2004), 378. 